\title{
Parity Games with Partial Information Played on Graphs of Bounded Complexity
}

\author{
Bernd Puchala ${ }^{1}$ and Roman Rabinovich ${ }^{2}$ \\ Mathematische Grundlagen der Informatik, RWTH Aachen University \\ \{puchala,rabinovich\}@logic.rwth-aachen.de
}

\begin{abstract}
We address the strategy problem for parity games with partial information and observable colors, played on finite graphs of bounded graph complexity. We consider several measures for the complexity of graphs and analyze in which cases, bounding the measure decreases the complexity of the strategy problem on the corresponding classes of graphs. We prove or disprove that the usual powerset construction for eliminating partial information preserves boundedness of the graph complexity. For the case where the partial information is unbounded we prove that the construction does not preserve boundedness of any measure we consider. We also prove that the strategy problem is ExPTIMEhard on graphs with directed path-width at most 2 and PSPACE-complete on acyclic graphs. For games with bounded partial information we obtain that the powerset construction, while neither preserving boundedness of entanglement nor of (undirected) tree-width, does preserve boundedness of directed path-width. Furthermore, if tree-width is bounded then DAGwidth of the resulting graph is bounded. Therefore, parity games with bounded partial information, played on graphs with bounded directed path-width or tree-width can be solved in polynomial time.
\end{abstract}

\section{Introduction}

Parity games are played by two players which move a token along the edges of a labeled graph by choosing appropriate edge labels, also called actions. The vertices of the graph, also called positions, have priorities and the winner of an infinite play of the game is determined by the parity of the least priority which occurs infinitely often. Parity games play a key role in modern approaches to verification and synthesis of state-based systems. They are the model-checking games for the modal $\mu$-calculus, a powerful specification formalism for verification problems. Moreover, parity objectives are a canonical form to express $\omega$-regular objectives and therefore capture fundamental properties of non-terminating reactive systems, cf. 18. Such a system can be modeled as a two-player game where changes of the system state correspond to changes of the game position. Situations where the change of the system can be controlled correspond to positions of player 0 ,

\footnotetext{
${ }^{1}$ Supported by the LINT project, ESF, www.esf.org

${ }^{2}$ Supported by the LINT and the GASICS projects, ESF, www.esf.org
} 
uncontrollable situations correspond to positions of player 1. A winning strategy for player 0 then yields a controller that forces the system into satisfying an $\omega$ regular specification.

The problem to determine, for a given parity game $G$ and a position $v$, whether player 0 has a winning strategy for $G$ from $v$, is called the strategy problem. The algorithmic theory of parity games with full information has received much attention during the past years, cf. [10. The most important property of parity games with full information is the memoryless determinacy which proves that the strategy problem for parity games is in NP $\cap$ co-NP.

However, assuming that both players have full information about the history of events in a parity game is not always realistic. For example, if the information about the system state is acquired by imprecise sensors or the system encapsulates private states which cannot be read from outside, then a controller for this system must rely on the information about the state and the change of the system to which it has access. I.e. in the game model, player 0 has uncertainties about the positions and actions in the game, so we have to add partial information to parity games in order to model this kind of problems. The uncertainties are represented by equivalence relations on the positions and actions in the game graph meaning that equivalent positions respectively actions are indistinguishable for player 0. Solving the strategy problem for such games is much harder than solving parity games with full information, since we have to keep track of the knowledge of player 0 during a play of the game. For this we compute, for any finite history, the set of positions that player 0 considers possible in this situation. This procedure is often referred to as powerset construction.

Such a knowledge tracking is inherently unavoidable and leads to an exponential lower bound for the time complexity of the strategy problem for reachability games with partial information [16] and a super-polynomial lower bound for the memory needed to implement winning strategies in reachability games [315. Therefore, it is expedient to look for classes of games with partial information, where the strategy problem has a lower complexity. A simple while effective approach is to bound the partial information in the game, i.e. the size of the equivalence classes of positions which model the uncertainties of player 0 about the current position. This is appropriate in situation where, e.g., the imprecision of the sensors or the amount of private information of the system does not grow if the system grows. Then, the game which results from the powerset construction has polynomial size, so partial information parity games with a bounded number of observable priorities can be solved in polynomial time. Hereby observable means, that the priorities are constant over equivalence classes. However, if the number of priorities is not bounded, we cannot prove this approach to be efficient, since the question whether full information parity games with arbitrarily many priorities can be solved in polynomial time is still open.

To obtain a class of parity games with partial information that can be solved in polynomial time, one has to bound certain other parameters. A natural approach is to bound the complexity of the game graphs with respect to appropriate measures. Such graph complexity measures have proven enormous usefulness in 
algorithmic graph theory. Several problems which are intractable in general can be solved efficiently on classes of graphs where such measures are bounded. The key note here is that bounded complexity with respect to appropriate measures allows to decompose the graph into small parts which are only sparsely related within the graph in a certain sense. One can then solve the problem on these small parts which requires, for each part, only a fixed amount of time, and combine the partial solutions in an efficient way. This has proven to be applicable to a large number of graph theoretic decision problems, e.g., all MSO-definable graph properties [5]. More recently, it has also been applied to the strategy problem for (full information) parity games. It has been shown that parity games played on graphs with bounded tree-width or bounded (monotone) DAG-width or bounded entanglement can be solved in polynomial time 24/13. The natural question is whether such results can also be obtained for games with partial information.

Since the direction of the edges is inherently important when solving games and when performing the powerset construction, we primarily consider measures for directed graphs. However, we prove a negative result about (undirected) tree-width, which is the most important measure for undirected graphs, as a prototype witness for the high potential of the powerset construction to create graph complexity when the direction of edges is neglected. From the large variety of measures for directed graphs we focus on DAG-width, directed path-width and entanglement. Two other important measures are directed tree-width [9] and Kelly-width [8]. For those measures, however, our techniques cannot be applied directly, due to somewhat inconvenient conditions in the definitions.

In Section 3 we prove that in the case where the partial information is unbounded, there are classes of graphs $G$ with complexity at most 2 such that the complexity of the corresponding powerset graphs is exponential in the size of $G$ for any measure we consider. We also prove that the strategy problem for reachability games with partial information is ExPTIME-hard on graphs with entanglement at most 2 and directed path-width at most 2 and that the problem is PSPACE-complete on acyclic graphs. Notice that reachability games form a subclass of parity games. Roughly speaking, these results show that bounding the graph complexity does not decrease the complexity of the strategy problem, as long as the partial information is unbounded.

In Section 4 we consider parity games with bounded partial information. In this case, the graphs which result from the powerset construction have polynomial size, so if the construction additionally preserves boundedness of appropriate graph complexity measure, then the corresponding strategy problem is in PTIME. For the case of tree-width and entanglement, however, we disprove this preservation of boundedness. However, if tree-width is bounded then DAG-width of the resulting graph is bounded as well. Finally, we prove that the construction does preserve boundedness of directed path-width and of non-monotone DAGwidth. So, parity games with bounded partial information, played on graphs of bounded directed path-width or bounded tree-width can be solved in polynomial time. Moreover, if DAG-width has bounded monotonicity cost, which is an open question, the same result holds for the case of bounded DAG-width. 


\section{Preliminaries}

Games and Strategies. A parity game has the form $G=\left(V, V_{0},\left(f_{a}\right)_{a \in A}, \mathrm{col}\right)$, where $V$ is the set of positions, $A$ is the set of actions and for each action $a \in A, f_{a}: \operatorname{dom}\left(f_{a}\right) \subseteq V \rightarrow V$ is a function. We write $v \stackrel{a}{\longrightarrow} w$ if $f_{a}(v)=w$. Furthermore, $V_{0} \subseteq V$ are the positions of player 0 and col $: V \rightarrow C$ is a function into a finite set $C \subseteq \mathbb{N}$ of colors (also called priorities). We define $V_{1}:=V \backslash V_{0}$ and $A_{i}:=\bigcup\left\{\operatorname{act}(v) \mid v \in V_{i}\right\}$ for $i=0,1$. The directed graph $(V, E)$ with $E=$ $\bigcup\left\{E_{a} \mid a \in A\right\}$ where $E_{a}=\left\{(u, v) \in V \times V \mid u \in \operatorname{dom}\left(f_{a}\right)\right.$ and $\left.f_{a}(u)=v\right\}$ for each $a \in A$ is called the game graph of $G$. Here we consider only finite games, i.e. games where $V$ and $A$ are finite.

For a finite sequence $\pi \in V(A V)^{*}$, by last $(\pi)$ we denote the last position in $\pi$. For $v \in V$, a play in $G$ from $v$ is a maximal finite or infinite sequence $\pi=v_{0} a_{0} v_{1} \ldots \in v(A V)^{*} \cup v(A V)^{\omega}$ such that $v_{i} \in \operatorname{dom}\left(f_{a_{i}}\right)$ and $f_{a_{i}}\left(v_{i}\right)=v_{i+1}$ for each $i$. A finite play $\pi$ is won by player 0 if last $(\pi) \in V_{1}$. An infinite play $\pi$ is won by player 0 if $\min \left\{c \in C \mid \operatorname{col}\left(v_{i}\right)=c\right.$ for infinitely many $\left.i<\omega\right\}$ is even. A play $\pi$ is won by player 1 if and only if it is not won by player 0 . A reachability game is a parity game with $C=\{1\}$, i.e. player 0 wins only finite plays which end in positions $v \in V_{1}$. Now let $\mathcal{H}_{\text {fin }}$ be the set of all finite histories $\pi \in V(A V)^{*}$ of plays in $G$ from $v$. A strategy for player $i$ for $G$ is a function $g:\left\{\pi \in \mathcal{H}_{\text {fin }} \mid \operatorname{last}(\pi) \in V_{i}\right\} \rightarrow A$ such that $g(\pi) \in \operatorname{act}($ last $(\pi))$ for all $\pi \in \operatorname{dom}(g)$. A history $\pi=v_{0} a_{0} v_{1} a_{1} v_{2} \ldots$ is called compatible with $g$ if for all $j$ such that $v_{j} \in V_{i}$ we have $a_{j}=g\left(v_{0} a_{0} \ldots a_{j-1} v_{j}\right)$. We call a strategy $g$ for player $i$ a winning strategy from $v_{0}$ if each play $\pi$ in $G$ from $v_{0}$ that is compatible with $g$ is won by player $i$.

Partial Information. The knowledge of player $i$ after some history $\pi \in \mathcal{H}_{\text {fin }}$ is given by an equivalence relation $\sim_{i} \subseteq \mathcal{H}_{\text {fin }} \times \mathcal{H}_{\text {fin }}$ where $\pi \sim_{i} \pi^{\prime}$ if $\pi$ and $\pi^{\prime}$ are indistinguishable for player $i$ by means of his given information. So, after $\pi$ has been played, to the best of player $i$ 's knowledge, it is possible that instead, $\pi^{\prime}$ has been played. A strategy $g:\left\{\pi \in \mathcal{H}_{\text {fin }} \mid \operatorname{last}(\pi) \in V_{i}\right\} \rightarrow A$ for player $i$ for $G$ is called a partial information strategy with respect to $\sim_{i}\left(\sim_{i}\right.$-strategy, for short $)$ if $g(\pi)=g\left(\pi^{\prime}\right)$ for all $\pi, \pi^{\prime} \in \mathcal{H}_{\text {fin }}$ with $\pi \sim_{i} \pi^{\prime}$. Notice that a $\sim_{i}$-strategy $g$ for player $i$ is winning from all positions in a set $U \subseteq V$ if and only if it is winning from a simulated initial position $v_{0}$ which belongs to player $1-i$ and from which he can secretly choose any position $v \in U$. Moreover, any $\sim_{i}$-strategy $g$ which is only defined on histories from some initial position $v_{0}$ can be extended to a $\sim_{i}$-strategy $g^{\prime}$ with $\operatorname{dom}(g)=\left\{\pi \in \mathcal{H}_{\text {fin }} \mid \operatorname{last}(\pi) \in V_{i}\right\}$ by giving $g^{\prime}$ appropriate value on histories from some initial position $v_{0}^{\prime} \neq v_{0}$. So in our antagonistic twoplayer setting, it suffices to consider strategies which are winning from single initial positions $v_{0}$ and only defined on histories from $v_{0}$.

Now, if we are given a game $G$, a position $v$ in $G$ and some equivalence relation $\sim_{i}$ on $\mathcal{H}_{\text {fin }}$, then the question whether player $i$ has a winning $\sim_{i}$-strategy for $G$ from $v$ is independent of the partial information of player $1-i$. Therefore, in this work we investigate games with partial information only for player 0 . 
We consider games played on finite graphs where player 0 has uncertainties about the positions and actions in the game, modeled by equivalence relations. The relation $\sim_{0}$ is then obtained by extending these equivalence relations to an equivalence relation on $\mathcal{H}_{\text {fin }}$. In particular, $\sim_{0}$ is finitely represented which is necessary when considering decision problems for games with partial information. A parity game with partial information has the form $\mathcal{G}=\left(G, \sim^{V}, \sim^{A}\right)$, where $G=\left(V, V_{0},\left(f_{a}\right)_{a \in A}\right.$, col $)$ is a parity game and $\sim^{V} \subseteq V \times V$ and $\sim^{A} \subseteq A \times A$ are equivalence relations such that the following conditions hold:

(1) If $u, v \in V$ with $u \sim^{V} v$ then $u, v \in V_{0}$ or $u, v \notin V_{0}$,

(2) If $a, b \in A_{0}$ with $a \neq b$ then $a \not{ }^{A} b$,

(3) If $u, v \in V_{0}$ with $u \sim^{V} v$, then $\operatorname{act}(u)=\operatorname{act}(v)$.

(4) If $u, v \in V$ with $u \sim^{V} v$ then $\operatorname{col}(u)=\operatorname{col}(v)$.

Condition (1) says that player 0 always knows when it is his turn and condition (2) says that player 0 can distinguish all the actions that are available to him at some position of the game. Condition (3) ensures that player 0 always knows which actions are available to him when it is his turn. Finally, condition (4) says that the colors of the game are observable for player 0 .

We say that a game $\mathcal{G}$ has bounded partial information, if there is some $k \in \mathbb{N}$, such that for any position $v \in V$ the equivalence class $[v]:=\left\{w \in V \mid v \sim^{V} w\right\}$ of $v$ has size at most $k$. Notice that the equivalence classes $[a]:=\left\{b \in A \mid a \sim^{A} b\right\}$ of actions $a \in A$ may, however, be arbitrarily large.

The equivalence relation on finite histories is defined as follows. For $\pi=$ $v_{0} a_{0} \ldots a_{n-1} v_{n}, \rho=w_{0} b_{0} \ldots b_{m-1} w_{m} \in V(A V)^{*}$, let

$$
\pi \sim^{*} \rho: \Longleftrightarrow n=m \text { and } v_{j} \sim^{V} w_{j} \text { and } a_{j} \sim^{A} b_{j} \text { for all } j .
$$

The winning region $\mathrm{Win}_{0}^{\mathcal{G}}$ of player 0 in $\mathcal{G}$ is the set of all positions $v \in V$ such that player 0 has a winning $\sim^{*}$-strategy for $\mathcal{G}$ from $v$.

Remark 1. Consider the interaction between components of a system where the behavior of each component is prescribed by a controller which has to rely on the information available to this component. In such settings it might seem more appropriate to ask for a $\sim_{0}^{*}$-strategy for player 0 which is winning against all $\sim_{1}^{*}$ strategies of player 1 rather than a winning $\sim_{0}^{*}$-strategy for player 0. However, it is easy to see that in our perfect recall setting, this is equivalent.

Powerset Construction. The usual method to solve games with partial information is a powerset construction originally suggested by John H. Reif in [16. The construction turns a game with partial information into a nondeterministic game with full information such that the existence of winning strategies for player 0 is preserved.

A nondeterministic parity game has the form $G=\left(V, V_{0},\left(E_{a}\right)_{a \in A}\right.$, col) where $V, V_{0}, A$, and col are as in a deterministic game and for $a \in A, E_{a}$ is a binary relation on $V$. Plays, strategies and winning strategies are defined as before. 
Nondeterministic games are not determined in general and hence not equivalent to deterministic games. However, for each nondeterministic game $G$ and each player $i \in\{0,1\}$, we can construct a deterministic game $G^{i}$ such that the existence of winning strategies for player $i$ is preserved. We simply resolve the nondeterminism by giving player $1-i$ control of nondeterministic choices. Technically, for any $v \in V$ and any $a \in \operatorname{act}(v)$ we add a unique $a$-successor of $v$ to the game graph which belongs to player $1-i$ and from which he can choose any $a$-successor of $v$ in the original game graph. The coloring of such a new position is the coloring of its unique predecessor. This construction does not increase the complexity of the game graph with respect to any measure we consider here.

Now for a parity game $\mathcal{G}=\left(G, \sim^{V}, \sim^{A}\right), G=\left(V, V_{0},\left(f_{a}\right)_{a \in A}, \mathrm{col}\right)$ with partial information, we construct the corresponding game $\bar{G}=\left(\bar{V}, \bar{V}_{0},\left(\bar{E}_{a}\right)_{a \in A}, \overline{\mathrm{col}}\right)$ with full information as follows. First, for $S \subseteq V$ and $B \subseteq A$ we define the set $\operatorname{Post}_{B}(S):=\left\{v \in V \mid \exists s \in S, \exists b \in B: b \in \operatorname{act}(s) \wedge f_{b}(s)=v\right\}$. The components of $\bar{G}$ are defined as follows.

$-\bar{V}=\left\{\bar{v} \in 2^{V} \mid \exists v \in V: \bar{v} \subseteq[v]\right\}$ and $\bar{V}_{0}=\bar{V} \cap 2^{V_{0}}$

$-\forall a \in A:(\bar{v}, \bar{w}) \in \bar{E}_{a}: \Longleftrightarrow \exists w \in \operatorname{Post}_{a}(\bar{v}): \bar{w}=\operatorname{Post}_{[a]}(\bar{v}) \cap[w]$

$-\operatorname{col}(\bar{v})=\operatorname{col}(v)$ for some $v \in V$.

It can be shown that this construction in fact preserves winning strategies for player 0 , that means, for any $v_{0} \in V$, player 0 has a winning $\sim^{*}$-strategy for $\mathcal{G}$ from $v_{0}$ if and only if he has a winning strategy for $\bar{G}$ from $\left\{v_{0}\right\}$. So when asking for a winning $\sim^{*}$-strategy for player 0 from a given position $v_{0}$, we are only interested in the part of the graph $\bar{G}$ which is reachable from $\left\{v_{0}\right\}$. We denote this subgraph of $\bar{G}$ by $\bar{G}_{v_{0}}$. The key-property for the correctness of the construction is given in the following lemma which is proved straightforwardly.

Lemma 2. For each finite history $\bar{\pi}=\bar{v}_{0} a_{1} \bar{v}_{1} \ldots a_{n} \bar{v}_{n}$ in $\bar{G}$ and all $v_{n} \in \bar{v}_{n}$, there is a finite history $\pi=v_{0} a_{1}^{\prime} v_{1} \ldots a_{n}^{\prime} v_{n}$ in $\mathcal{G}$ such that $v_{i} \in \bar{v}_{i}$ for all $i \in$ $\{0, \ldots, n\}$ and $a_{i}^{\prime} \sim^{A} a_{i}$ for all $i \in\{1, \ldots, n\}$.

Graph Complexity. We consider only directed graphs without multi-edges, but possibly with self-loops, i.e. a graph is a pair $G=(V, E)$ where $E \subseteq V \times V$. An undirected graph is a graph with a symmetric edge relation.

All measures we consider can be characterized in terms of cops and robber games, where several cops try to catch a robber on a graph. Technically, these games are reachability games. We do not give formal descriptions of the games but merely describe them in an informal way. In a graph searching game there are two players, a cop player and a robber player. Basically, the robber player moves a robber token along cop free paths of the graph. The cop player has a number $k$ of cops at his disposal and he can place and move them on and between vertices. At the very moment a cop is moving he does not block any vertex. The goal of the cop player is to place a cop on the robber, the robber player's goal is to elude capture. That means, infinite plays are won by the robber and finite plays, which end in a position where the robber has no legal moves available, are 
won by the cops. The number $k$ of cops is a parameter of the game, that means, for any natural number $k$ we have a $k$-cops and robber game.

Tree-width, see [17, denoted tw, is a measure defined for undirected graphs and the tree-width of a directed graph is the tree-width of his symmetric closure. In the tree-width game, the cops can be placed and moved in the graph arbitrarily. When a new set $U$ of at most $k$ vertices is chosen to be occupied by the cops, the robber may move along any cop-free path, i.e. no vertex on this path which has been occupied by a cop is also occupied by a cop according to $U$. The robber may move at unlimited speed, i.e. he may move along a whole path in one step.

DAG-width, introduced in 214 and denoted dw, is a generalization of tree-width to directed graphs. The DAG-width game has exactly the same rules as the tree-width game with the only difference that now, the robber has to respect the direction of the edges. The directed path-width, denoted dpw, is defined by the same game where now, the robber is invisible. So, a strategy for the cop player has to yield the same decision in any two situations which differ only in the current position of the robber.

Finally, in the entanglement-game [4] the cop player may, in each round, do nothing or place one of the $k$ cops on the current position of the robber. No matter what the cops do, the robber must go from his recent vertex to a new vertex, which is not occupied by a cop, along an edge of the graph.

For $X \in\{\mathrm{tw}, \mathrm{dw}, \mathrm{dpw}\}, X(G)$ is the least natural number $k$ such that $k+1$ cops $(k$ cops, if $X=\mathrm{dw})$ have a (robber-)monotone winning strategy for the $X$ game on $G$. A strategy $f$ for the cops is called (robber-)monotone, if in any play compatible with $f$, the robber can never reach any vertex that has previously been unavailable for him. Such a monotone winning strategy for $k$ cops yields a decomposition of $G$ into (possibly complex) parts of size at most $k$ which are only sparsely related among each other. Such decompositions often allow for efficient dynamic solutions of hard graph problems. Notice that clearly, $\operatorname{dw}(G) \leq$ $\operatorname{dpw}(G)+1$ for any graph $G$.

The entanglement of a graph $G$, denoted ent $(G)$, is the least natural number $k$ such that $k$ cops have an arbitrary winning strategy for the entanglement game on $G$. For entanglement, only for $k=2$, a decomposition in the above sense is known [6]. Nevertheless, parity games can be solved efficiently on graph classes of bounded entanglement.

In the following, let $\mathcal{M}=\{\mathrm{tw}, \mathrm{dw}, \mathrm{dpw}$, ent $\}$. We say that a measure $X \in \mathcal{M}$ has monotonicity cost at most $f$ for a function $f: \mathbb{N} \rightarrow \mathbb{N}$ if, for any graph $G$ such that $k$ cops have a winning strategy for the $X$-game on $G, k+f(k)$ cops have a monotone winning strategy for the $X$-game on $G$. We say that $X$ has bounded monotonicity cost if there is a function $f: \mathbb{N} \rightarrow \mathbb{N}$ such that $X$ has monotonicity cost at most $f$. Tree-width has monotonicity cost 0 [17] and the same holds for directed path-width, 117. On the contrary, DAG-width does not have monotonicity cost 0 [12. Whether DAG-width has bounded monotonicity cost, is an important open problem in structure theory of directed graphs. 


\section{Unbounded Partial Information}

First, when the partial information is unbounded, it is easy to prove that boundedness of graph complexity measures is not preserved by the powerset construction and does not prevent the size of the graph to grow exponentially. We show that even the measures themsleves grow exponentially.

Before we prove the first result of this section we note the well-known fact that, for any $n \in \mathbb{N}$, we have $X\left(\mathfrak{G}_{\mathfrak{n}}\right) \geq n$ for all $X \in \mathcal{M}$, where $\mathfrak{G}_{n}$ is full, undirected $n \times n$-grid defined as follows: $\mathfrak{G}_{n}=\left(V_{n}, E_{n}\right)$ with $V_{n}=\{(i, j) \mid 1 \leq$ $i, j \leq n\}$ and $\left(\left(i_{1}, j_{1}\right),\left(i_{2}, j_{2}\right)\right) \in E \Leftrightarrow i_{1}=i_{2}$ and $\left|j_{1}-j_{2}\right|=1$, or $i_{1}=i_{2}$ and $\left|j_{1}-j_{2}\right|=1$.

Proposition 3. There are games $\mathcal{G}_{n}, n \in \mathbb{N}$ with partial information and with $X\left(\mathcal{G}_{n}\right) \leq 2$ for all $n \in \mathbb{N}$ and for any $X \in \mathcal{M}$, such that the powerset graphs $\bar{G}_{n}$ have exponential measure $X$ in the size of $\mathcal{G}_{n}$ for any $X \in \mathcal{M}$.

Proof. From a fairly simple graph, we generate a graph containing an undirected square grid of exponential size as a subgraph. This is possible because we can consider large equivalence classes of positions and actions.

Consider a disjoint union of $n$ directed cycles of length 2 with self-loops on each vertex where any two positions are equivalent. Additionally we have an initial position such that, by applying the powerset construction from this position, we obtain a set which contains exactly one element from each cycle. Continueing, we get sets that represent binary numbers with $n$ digits and for each digit we have an action which causes exactly this digit to flip. So, using the Gray-code, we can create all binary numbers with $n$ digits by successively flipping each digit. If we do this independently for the first $n / 2$ digits and for the last $n / 2$ digits, it is easy to see that the resulting positions are connected in such a way, that they form an undirected grid $\bar{G}_{n}$ of size $2^{n / 2} \times 2^{n / 2}$, for which we have $X\left(\bar{G}_{n}\right) \geq 2^{n / 2}$ for any measure $X \in \mathcal{M}$.

To be more precise, for even $n<\omega$, let $\mathcal{G}_{n}=\left(G_{n}, \sim_{n}^{V}, \sim_{n}^{A}\right)$, where $G_{n}=$ $\left(V_{n}, \emptyset,\left(f_{a}^{n}\right)_{a \in A_{n}}\right)$ is the following game graph. The set of vertices is $\left\{v_{0}\right\} \cup$ $\{(i, j) \mid j \in\{0,1\}, 1 \leq i \leq n\}$ where $i$ stands for the number of the cycle and $j$ for the number of a vertex in the cycle. The actions are $A_{n}=\left\{a_{i} \mid 1 \leq i \leq\right.$ $n\} \cup\left\{\neg_{i} \mid 1 \leq i \leq n\right\}$. Here the actions $a_{i}$ lead from $v_{0}$ to the cycles: we have $v_{0} \stackrel{a_{i}}{\longrightarrow}(0, i)$ for $1 \leq i \leq n$. Further actions make the cycles:

$-(i, j) \stackrel{\neg_{i}}{\longrightarrow}(i, 1-j)$ for $1 \leq i \leq n$ and $j \in\{0,1\}$.

$-(i, j) \stackrel{\neg k}{\longrightarrow}(i, j)$ for $1 \leq i \leq n$ with $k \neq i$ and $j \in\{0,1\}$.

Partial information is defined by $(i, j) \sim_{n}^{V}(k, l)$ and $a_{i} \sim_{n}^{A} a_{k}$ for any $1 \leq$ $i, j, k, l \leq n$. So each two positions from any two cycles are indistinguishable and each two of the actions $a_{\sigma}^{i}$ are indistinguishable. It is clear that $X\left(G_{n}\right) \leq 3$ for any measure $X \in \mathcal{M}$. 
In Figure 1 the graph $\mathcal{G}_{2}$ and the powerset graph $\bar{G}_{v_{0}}^{n}$ are depicted. The position $\left\{v_{0}\right\}$ of the powerset graph is omitted and a position $(i, j)$ is represented as $j$. Positions $\left\{j_{1}, j_{2}\right\}$ are denoted $j_{1} j_{2}$.

Now, performing the powerset construction on $\mathcal{G}_{n}$ from $v_{0}$ we obtain the graph $\bar{G}_{v_{0}}^{n}$ which obviously contains the position $\{(1,0), \ldots,(n, 0)\}$. From this position, an undirected square grid of exponential size is constructed as follows. We successively apply actions $\neg_{i}$ for $i \in\{1, \ldots, n / 2\}$ to create each vertex $\left\{\left(1, j_{1}\right), \ldots,\left(n / 2, j_{n / 2}\right),(1,0), \ldots,(n, 0)\right\}$ with $j_{1}, \ldots, j_{n / 2} \in\{0,1\}$. In each step we can change exactly one $j_{r}$ to $1-j_{r}$, so the creation of all these vertices from $\{(1,0), \ldots,(n / 2,0),(n / 2+1,0), \ldots,(n, 0)\}$ can, for instance, be done using the usual Gray-code for binary numbers: we get the next vertex by applying $\neg_{i}$ to the previous vertex $\left\{\left(1, j_{1}\right), \ldots,\left(n / 2, j_{n / 2}\right),(n / 2+1,0), \ldots,(n, 0)\right\}$, which changes exactly one position $\left(i, j_{i}\right)$. This undirected path forms the upper horizontal side of the grid. Analogously, by successively applying the actions $\neg^{i}$ for $i \in\{n / 2+1, \ldots, n\}$ we can create each vertex $\{(1,0), \ldots,(n / 2,0)$, $\left.\left(n / 2+1, j_{n / 2+1}\right), \ldots,\left(n, j_{n}\right)\right\}$ with $j_{n / 2+1}, \ldots, j_{n} \in\{0,1\}$ using the Gray-code. This undirected path forms the left vertical side of the grid. (Of course, terms like left and horizontal are used here only for convenience, they do not have any mathematical meaning in this context.)

Likewise, given any vertex $\left\{\left(1, j_{1}\right), \ldots,\left(n / 2, j_{n / 2}\right),(n / 2+1,0), \ldots,(n, 0)\right\}$ we can create any vertex $\left\{\left(1, j_{1}\right), \ldots,\left(n / 2, j_{n / 2}\right),\left(n / 2+1, j_{n / 2+1}\right), \ldots,\left(n, j_{n}\right)\right\}$ by successively applying the actions $\neg_{i}$ for $i \in\{n / 2+1, \ldots, n\}$ in the same order as before and given any vertex $\left\{(1,0), \ldots,(n / 2,0),\left(n / 2+1, j_{n / 2+1}\right), \ldots,\left(n, j_{n}\right)\right\}$, by successively applying the actions $\neg_{i}$ for $i \in\{1, \ldots, n / 2\}$, we can create any vertex $\left\{\left(1, j_{1}\right), \ldots,\left(n / 2, j_{n / 2}\right),\left(n / 2+1, j_{n / 2+1}\right), \ldots,\left(n, j_{n}\right)\right\}$. All these paths form a $2^{n / 2} \times 2^{n / 2}$-grid and therefore, the tree-width of $\bar{G}_{v_{0}}^{n}$ is exponential in the size of $\mathcal{G}_{n}$. Furthermore, using that $\bar{G}_{v_{0}}^{n}$ is undirected one easily checks that for all $X \in \mathcal{M}, X\left(\bar{G}_{v_{0}}^{n}\right) \geq \operatorname{tw}\left(\bar{G}_{v_{0}}^{n}\right)$.

Remark 4. Notice that exponential size of the resulting graph is not needed for unbounded growth of graph complexity measures. If we consider, for example, a disjoint union of two undirected paths of length $n$ with appropriate actions and self-loops on all positions, then the construction of the corresponding powerset graph yields an $n \times n$-grid.

Towards our analysis of the complexity of the strategy problem for games with partial information on graphs of bounded complexity, we first note that on trees, solving games with partial information is not harder than solving games with full information. Performing the powerset construction on a tree, we again obtain a tree, where the set of positions on each level partitions the set of positions on the corresponding level of the original tree. This new tree can therefore be computed in polynomial time and has at most as many vertices as the original tree. In the following results we prove that at soon as we consider at least DAGs which are not trees, the strategy problem for reachability games becomes intractable as long as we do not bound any other parameters. 

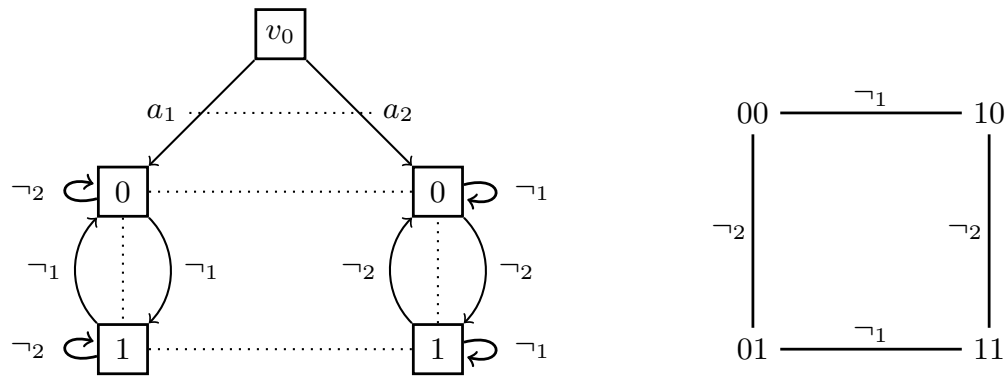

Fig. 1. Game graph $\mathcal{G}_{2}$ and the powerset graph $\bar{G}_{v_{0}}^{2}$.

For the proofs of the subsequent results, we need the following facts, see for example [19.

\section{Lemma 5.}

(1) APSPACE $=$ EXPTIME.

(2) For all $L \in \operatorname{AsPACE}(S(n))$ with $S(n) \geq n$ there is an alternating Turing machine with a single tape and space bound $S(n)$ which accepts $L$.

(3) APTIME $=$ PSPACE.

(4) For all $L \in \operatorname{Atime}(T(n))$ with $T(n) \geq n$ there is an alternating Turing machine with a single tape and time bound $O\left(T^{2}(n)\right)$ which accepts $L$.

Theorem 6. The following problem is ExPTIME-hard. Given a partial information reachability game $\mathcal{G}=\left(G, \sim^{V}, \sim^{A}\right)$ with $\operatorname{ent}(G) \leq 2$ and $\operatorname{dpw}(G) \leq 3$ and a position $v_{0} \in V(G)$, is $v_{0} \in \mathrm{Win}_{0}^{\mathcal{G}}$ ?

Proof. By Lemma 5, for any $L \in$ Exptime, there is an alternating Turingmachine $M=\left(Q, \Gamma, \Sigma \supseteq \Gamma, q_{0}, \delta\right)$ with only one tape and space bound $n^{k}$ for some $k \in \mathbb{N}$, where $n$ is the size of the input, that recognizes $L$. As usual, $Q$ is the set of states, $\Gamma$ and $\Delta$ are the input and the tape alphabets, $q_{0}$ is the initial state, and $\delta$ is the transition relation. First assume that $M$ is deterministic. We describe the necessary changes to prove the general case afterwards.

Let $\Delta=\Sigma \uplus(Q \times \Sigma) \uplus\{\#\}$. Then each configuration $C$ of $M$ is described by a word $C=\# w_{0} \ldots w_{i-1}\left(q w_{i}\right) w_{i+1} \ldots w_{t} \# \in \Delta^{*}$ over $\Delta$ and since $M$ has space bound $n^{k}$ and we have $k \geq 1$, w.l.o.g. we can assume that $|C|=n^{k}+2$ for all configurations $C$ of $M$ on inputs of length $n$. Moreover, for a configuration $C$ of $M$ and some $2 \leq i \leq n^{k}+1$ the symbol number $i$ of $C^{\prime}$ where $C^{\prime}=\operatorname{Next}(C)$ only depends on the symbols number $i-1, i$ and $i+1$ of $C$. So there is a function $f: \Delta^{3} \rightarrow \Delta$ such that for any configuration $C$ of $M$ and any $2 \leq i \leq n^{k}+1$, if the symbols number $i-1, i$ and $i+1$ of $C$ are $a_{-1} a_{0} a_{1}$ then the symbol number $i$ of the successor configuration $C^{\prime}=\operatorname{Next}(C)$ of $C$ is $f\left(a_{-1} a_{0} a_{1}\right)$.

Now let $u=u_{1} \ldots u_{n} \in \Gamma^{*}$. The idea for the game corresponding to $u$ is the following. Player 0 selects symbols from $\Delta$, such that the sequence constructed in this way forms an accepting run of $M$ on $u$. In order to check the correctness of the construction that player 0 provides, player 1 may, at any point during 
the play but only once, memorize the recent position $i \in\left\{1, \ldots, n^{k}\right\}$ within the recent configuration and the last three symbols chosen by player 0 . Then, in the next configuration, player 1 may check the $i$-th symbol chosen by player 0 to be correct according to the symbols which he has previously memorized and the function $f$. If the $i$-th symbol proves incorrect, player 0 loses, otherwise, player 1 loses. Player 0 must not notice when player 1 memorizes the recent position, which defines the partial information in the game. To justify the bounds on the graph complexity measures that we have claimed, we define the game formally.

We define the game $\mathcal{G}_{u}=\left(G, \sim^{V}, \sim^{A}\right)$ with partial information as follows. The set of positions is $V=\left\{v_{0}\right\} \cup\{0,1\} \times \Delta \times\left\{0, \ldots, n^{k}\right\} \times Q \times\left\{0, \ldots, n^{k}\right\} \times \Delta^{3}$, so a position has the form $\left(\sigma, \delta, i, q, j, \delta_{1} \delta_{2} \delta_{3}\right)$ where $\sigma$ is the player whose turn it is, $\delta$ is the recent symbol as chosen by player 0 and $i$ is the recent position within the recent configuration. Moreover, $q$ is the last state $q \in Q$ chosen by player 0 . Finally, $j$ and the sequence $\delta_{1} \delta_{2} \delta_{3}$ represent the information which player 1 has memorized. Now we give a complete list of the moves that can be made in the game. For convenience, the actions are omitted in the description. The player whose turn it is, is given by the first component of a position except for position $v_{0}$ which belongs to player 1 . Moreover, for an $n$-tuple $\bar{x}=\left(x_{1}, \ldots, x_{n}\right)$ we denote $x_{i}$ by $\operatorname{pr}_{i}(\bar{x})$. The possible moves are:

- from $v_{0}$ to $\left(0, \#, 0, q_{0}, j, \delta_{1} \delta_{2} \delta_{3}\right)$ where $j \neq \emptyset$ and $\delta_{1} \delta_{2} \delta_{3}$ are symbols number $j-1, j$ and $j+1$ of the initial configuration $C_{\text {in }}(u)$ of $M$ on $u$

- from $v_{0}$ to $\left(0, \#, 0, q_{0}, 0, \# \# \#\right)$

- from $\left(0, \delta, i, q, 0, \delta_{1} \delta_{2} \delta\right)$ with $i \leq n^{k}$ to $\left(1, \delta^{\prime}, i+1, q^{\prime}, 0, \delta_{2} \delta \delta^{\prime}\right)$ where $\delta^{\prime} \in \Delta \backslash\{\#\}$ and $q^{\prime}=q$ if $\delta^{\prime} \notin Q \times \Sigma$ and $q^{\prime}=\operatorname{pr}_{1}\left(\delta^{\prime}\right)$ if $\delta^{\prime} \in Q \times \Sigma$

- from $\left(0, \delta, n^{k}+1, q, 0, \delta_{1} \delta_{2} \delta\right)$ to $\left(1, \#, n^{k}+2, q, 0, \delta_{2} \delta \#\right)$

- from $\left(0, \delta, i, q, j, \delta_{1} \delta_{2} \delta_{3}\right)$ with $i \neq n^{k}+1$ and $j \neq 0$ to $\left(1, \delta^{\prime}, i+1, q, j, \delta_{1} \delta_{2} \delta_{3}\right)$ where $\delta^{\prime} \in \Delta \backslash\{\#\}$

- from $\left(0, \delta, n^{k}+1, q, j, \delta_{1} \delta_{2} \delta_{3}\right)$ with $j \neq 0$ to $\left(0, \#, n^{k}+2, q, j, \delta_{1} \delta_{2} \delta_{3}\right)$.

- from $\left(1, \delta, i, q, j, \delta_{1} \delta_{2} \delta_{3}\right)$ with $i \neq j$ to $\left(0, \delta, i, q, j, \delta_{1} \delta_{2} \delta_{3}\right)$.

- from $\left(1, \delta, i, q, 0, \delta_{1} \delta_{2} \delta\right)$ to $\left(0, \delta, i, q, i-1, \delta_{1} \delta_{2} \delta\right)$, if $i \geq 3$.

- $\operatorname{from}\left(1, \#, n^{k}+2, q, 0, \delta_{1} \delta_{2} \#\right)$ with $q \notin Q_{\text {acc }} \cup Q_{\text {rej }}$, to $(0, \#, 0,0, \# \# \#)$.

- from $\left(1, \delta, n^{k}+2, q, j, \delta_{1} \delta_{2} \delta_{3}\right)$ with $j \neq 0$ to $\left(0, \#, 0, j, \delta_{1} \delta_{2} \delta_{3}\right)$.

Of a position $\left(\sigma, \delta, i, q, j, \delta_{1} \delta_{2} \delta_{3}\right)$, only the first three entries are visible to player 0 , that means, two positions are indistinguishable if and only if the they coincide in the first three components. Which amounts exactly to the claim that player 0 is never aware whether his construction is checked by player 1 . Moreover, any two actions of player 1 are indistinguishable for player 0 . Now, at positions $\left(1, \delta, i, q, i, \delta_{1} \delta_{2} \delta_{3}\right)$, player 0 has won if $f\left(\delta_{1} \delta_{2} \delta_{3}\right)=\delta$, otherwise, player 1 has won. At a position $\left(1, \#, n^{k}+2, q, 0, \delta_{1} \delta_{2} \#\right)$ with $q \in Q_{\text {acc }} \cup Q_{\text {rej }}$, player 0 has won if $q \in Q_{\text {acc }}$ and player 1 has won, if $q \in Q_{\text {rej }}$.

Structurally, $G$ consists of $|\Delta|^{3} \cdot n^{k}+1$ augmented DAGs $t_{0}$ and $t_{j}^{\bar{\delta}}, j=$ $1, \ldots, n^{k}, \bar{\delta} \in \Delta^{3}$. Each $t_{j}^{\bar{\delta}}$ has a unique top node, edges from any non-bottom 
level only to the level below and $2 \cdot n^{k}+1$ levels in total. For a fixed number $j \in\left\{1, \ldots, n^{k}\right\}$, we refer to the union of the $t_{j}^{\bar{\delta}}$ for $\bar{\delta} \in \Delta^{3}$ by $t_{j}$. We also have a unique root node $v_{0}$ for the whole graph from which there is an edge to the top of $t_{0}$ and, for any $0<j \leq n^{k}$, there is an edge to exactly one top node of $t_{j}$. Moreover, for any $0 \leq j \leq n^{k}$ and any $\bar{\delta} \in \Delta^{3}$, from any node at level $2 \cdot n^{k}+1$ of $t_{j}$, there is a back-edge to the top of $t_{j}$ (which is the only cyclicity in the graph). Finally, on the $i$-th level of $t_{i}$ for $i \geq 1$, there are no outgoing edges. So obviously $\operatorname{ent}(G) \leq 1$ and $\operatorname{dpw}(G) \leq 2$. (Notice that we are still considering the special case where $M$ is deterministic.)

Now, as long as player 1 has not yet decided to memorize, the play takes place in $t_{0}$ and player 1 keeps track of the information which he needs in case he decides to memorize. If he decides to memorize at position $i$ within some configuration, then this means, that he wants to check the character at position $i-1$ within the next configuration, given the characters $i-2, i-1$ and $i$ of the current configuration, so he switches to the corresponding position in $t_{i-1}$. As we have already mentioned, player 0 never notices whether player 1 leaves $t_{0}$ or not. If player 1 lets player 0 write down characters until some character $(q, a)$ with $q \in Q_{\text {acc }} \cup Q_{\text {rej }}$ is written, then the winner is determined according to the state $q$. (Notice that player 0 finally has to write a character $(q, a)$ with $q \in Q_{\text {acc }} \cup Q_{\text {rej }}$ since he has a reachability objective.) If player 1 wants to check player 0's construction, then he can decide to do this at exactly one point during a play by moving to some $t_{i}$ with $i \neq 0$ as mentioned above. If the character he wants to check is incorrect he wins, otherwise he loses.

Obviously, $\mathcal{G}$ can be constructed from a given input $u \in \Gamma^{*}$ in polynomial time. If the word $u$ is accepted by $M$, then clearly player 0 wins the game from $v_{0}$ by simply writing down the run of $M$ on $u$ character by character. Now let conversely $f$ be a winning strategy for player 0 for $\mathcal{G}$ from $v_{0}$ and let $\bar{\delta}=\delta_{1} \delta_{2} \ldots \delta_{k}$ be the sequence of characters given by player 0 according to $f$, if player 1 plays in $t_{0}$ all the time. Assume, $\bar{\delta}$ does not represent the unique run of $M$ on $u$. Then there is some $i<k$, such that, up to position $i, \bar{\delta}$ coincides with the unique run of $M$ on $u$, but up to position $i+1$ it does not. So, let $\delta_{i+1}$ be the $l$-th position within the recent configuration. We modify the play as follows. During the construction of the previous configuration (or from $v_{0}$, if the previous configuration is the initial configuration), player 1 chooses a position of the form $\left(0, \delta, i+2, q, i+1, \delta_{1} \delta_{2} \delta\right)$, i.e. he memorizes at position $i+2$. By our assumption on $\bar{\delta}$, the resulting play is lost by player 0 . However, since player 0 does not notice that player 1 memorizes and $f$ is a partial information strategy, the resulting play is compatible with $f$ in contradiction to the fact that $f$ is a winning strategy for player 0 for $\mathcal{G}$ from $v_{0}$. So, in contrary to our assumption, $\bar{\delta}$ represents the unique run of $M$ on $u$. Due to the definition of the winning condition of $\mathcal{G}$ this run must be accepting, i.e. $u \in L(M)$.

Now consider the general case, where $M$ is not necessarily deterministic. W.l.o.g. we can assume that each non-terminal configuration of $M$ has exactly two successor configurations. If there is a configuration $C$ with just a single successor configuration then we add a default successor to $C$ which leads to ac- 
ceptance if $C$ is universal and which leads to rejectance if $C$ is existential. If there is a configuration with $b>2$ successors, then we replace this $b$-branching configuration tree by a binary branching configuration tree of depth $b$ by modifying the transition function of $M$ in an appropriate way. Obviously, this construction can be done in such a way that it merely increases the state space of $M$ and the time bound by a constant factor, but not the space bound. Now, instead of one function $f$, we have two functions $f_{1}, f_{2}: \Delta^{3} \rightarrow \Delta$, such that the following holds. If $C$ is a configuration of $M, l \in\{1,2\}$ and $2 \leq i \leq n^{k}+1$, and the symbols number $i-1, i$ and $i+1$ of $C$ are $a_{-1} a_{0} a_{1}$ then the symbol number $i$ of the successor configuration $C_{l}=\operatorname{Next}_{l}(C)$ number $l$ of $C$ is $f_{l}\left(a_{-1} a_{0} a_{1}\right)$. Thus, for each $j \in\left\{0, \ldots n^{k}\right\}$ we use two copies $t_{j}^{1}$ and $t_{j}^{2}$ of $t_{j}$. From $v_{0}$, for all $l \in\{0,1\}$, an edge to exactly one top node of $t_{j}^{l}$ exists. At a leaf node of $t_{j}^{l}$, if the recent configuration is existential (as determined by the recent state) then player 0 chooses whether to proceed at a top node of $t_{j}^{1}$ or of $t_{j}^{2}$. If the recent configuration if universal, then player 1 makes this choice. (Notice that the particular top node of $t_{i}^{l}$ which is chosen is determined by the recent position, it is merely the $l$ which is chosen by one of the players.) Partial information is defined as before with the additional condition that player 0 observes the copy of $t_{j}$ in which the play currently takes place. Now, for $i>0$ and $l \in\{1,2\}$, in $t_{i}^{l}$ the correctness of the construction player 0 provides is checked using the function $f_{l}$. The trick which player 1 uses to find the flaw in the construction if $M$ does not accept some input $u$ is exactly the same as before. Clearly these modifications merely increase the entanglement of the graph from at most 1 to at most 2. and the directed path-width from at most 2 to at most 3 .

Remark 7. It easy to see, that the tree-width of the game graphs constructed in the proof of Theorem 6 is bounded by some $k \in \mathbb{N}$ which is independent of the input $u$. Therefore, the strategy problem for reachability games with partial information on graphs of tree-width at most $k$ is ExPTIME-hard.

Remark 8. Notice that the graph which we have constructed in the proof of Theorem 6 is not strongly connected and that partial information cuts through different strongly connected components. However, to make the graphs strongly connected it suffices to connect each position of player 1 via an undirected edge with some dummy position $\diamond$ which belongs to player 0 and from which he can choose to go to a terminal position of player 1 immediately. This merely increases both entanglement and directed path width by just 1 and obviously does not harm the correctness of the construction.

The cases of entanglement and directed path-width at most 1 are still open. However, the strategy problem for sequence-forcing games with partial information, where player 0 tries to enforce a certain sequence of fixed length of positions, is ExPTIME-hard on graphs of entanglement and directed path-width at most 1. Sequence-forcing games can be polynomially reduced to reachability games so we obtain, roughly speaking, the following result. 
Theorem 9. Adding partial information to games played on graphs of entanglement at most and directed path-width at most 1 can cause an unavoidable exponential blow-up of the time complexity of the corresponding strategy problem.

Proof. We consider sequence-avoidance conditions which have the form $(S, \mathrm{col})$ where col : $V \rightarrow C$ is a coloring of $V$ and $S \subseteq\{1, \ldots, r\}^{k}$ is a set of forbidden sequences of length $k$ for some $k<\omega$. Player 0 wins an infinite play $\pi$ of a sequenceavoidance game if for all $i<\omega$ we have $\operatorname{col}(\pi(i)) \operatorname{col}(\pi(i+1)) \ldots \operatorname{col}(\pi(i+k)) \notin S$. It is not hard to see that if $k$ is fixed, sequence-avoidance games can be polynomially reduced to safety games by using a memory which stores the last $k$ colors that have occurred. (Notice that this reduction may, however, increase the complexity of the game graph.) In particular, the strategy problem for sequenceavoidance games with fixed $k$ is in PTIME. Here, $k=3$ suffices.

Now we modify the proof of Theorem 6 as follows. From the nodes on level $2 \cdot n^{k}+1$ of $t_{j}^{1}$ and $t_{j}^{2}$ we do not allow moves directly back to the top of $\left(t_{j}^{\bar{\delta}}\right)^{1}$ or $\left(t_{j}^{\bar{\delta}}\right)^{2}$, but we redirect all edges to a single position $(\bar{\delta}, j)$, which belongs to player 0 . From this position, player 0 may move to position $(0, \bar{\delta}, j)$ which belongs to player 0 or to position $(1, \bar{\delta}, j)$ which belongs to player 1 . Furthermore, from $(0, \bar{\delta}, j)$, player 0 chooses whether to proceed in $\left(t_{j}^{\bar{\delta}}\right)^{1}$ or in $\left(t_{j}^{\bar{\delta}}\right)^{2}$ and from $(1, \bar{\delta}, j)$ player 1 makes this choice. Partial information is defined as before, so all the positions $(\bar{\delta}, j)$ are indistinguishable for player 0 and two positions $(\sigma, \bar{\delta}, j)$ and $\left(\sigma^{\prime}, \bar{\delta}^{\prime}, j^{\prime}\right)$ are distinguishable for player 0 if and only if $\sigma \neq \sigma^{\prime}$. The coloring of the positions is defined as follows. The nodes on level $2 \cdot n^{k}+1$ of $t_{j}^{1}$ and $t_{j}^{2}$ are colored with 0 , if the recent configuration is existential (as determined by the recent state) and with 1 , if the recent configuration if universal. Each position $(\bar{\delta}, j)$ is colored with 0 , the positions $(0, \bar{\delta}, j)$ get the color -1 and the positions $(1, \bar{\delta}, j)$ get the color 1 . All other positions are colored with 0 . Now, $S=\{(1,0,-1)\}$, i.e. the unique sequence that player 0 wants to avoid is $(1,0,-1)$. This forces player 0 into giving control back to player 1 if the last configuration that he has constructed has been existential. Now the proof of Theorem 6 carries over without essential modifications, showing that the strategy problem for sequenceavoidance games, played on graphs of entanglement at most 1 and directed pathwidth at most 2 is ExPTIME-hard.

Finally, if the we consider acyclic game graphs, the strategy problem for partial information reachability games is PSPACE-complete. Notice that acyclic graphs are precisely those having DAG-width 1.

Theorem 10. The strategy problem for reachability games with partial information on acyclic graphs is PSPACE-complete.

Proof. Let the given game be $\mathcal{G}=\left(G, \sim^{V}, \sim^{A}\right)$ and let $v_{0}$ be the initial position. First we prove the membership in PsPace. The idea is that carrying out the powerset construction on an acyclic graph $G$ we again obtain an acyclic graph $\bar{G}$ where by Lemma 2 the paths in $\bar{G}$ are not longer than the paths in $G$. So, 
we can solve the reachability game on $\bar{G}$ by an APtime algorithm. According to Lemma 5 it suffices to construct an alternating algorithm with polynomial time bound for the problem. We describe this algorithm informally, claiming that its correctness is obvious. Starting from $\left\{v_{0}\right\}$, we proceed as follows. Given a position $\bar{v} \in \bar{V}$ in the corresponding game $\bar{G}_{v_{0}}$ with full information, if $\bar{v} \in \bar{V}_{0}$, then an existential step is performed in which a successor position of $\bar{v}$ is guessed. If $\bar{v} \in \bar{V}_{1}$, then a universal step is performed in which a successor position of $\bar{v}$ is chosen. If the computation reaches a leaf-node in $\bar{V}_{1}$ the algorithm accepts and if the computation reaches a leaf-node in $\bar{V}_{0}$ the algorithm rejects. The construction of a successor position of some position $\bar{v}$ can obviously be done in polynomial time. Moreover, if $\bar{\pi}=\bar{v}_{0} \rightarrow \bar{v}_{1} \rightarrow \ldots \rightarrow \bar{v}_{k}$ is any path in $\bar{G}_{v_{0}}$ then, according to Lemma 2 , there is a path $\pi=v_{0} \rightarrow v_{1} \rightarrow \ldots \rightarrow v_{k}$ with $v_{i} \in \bar{v}_{i}$ for $i=0, \ldots, k$. Since $G$ is acyclic, $k \leq n$. So, the computation stops after at most $n$ steps.

Conversely, if the machine from the proof of Theorem 6 is a PTIME ATM, we do not insert edges back to the roots of the DAGs $t_{i}$ but we go to the roots of new copies of the DAGs $t_{i}$. Since $M$ is polynomially time bounded, the resulting graph has polynomial size and is, by construction, acyclic.

To prove the hardness more formally let $L \in$ PSPACE be any PSPACE problem. Then, according to Lemma 5, there is an alternating Turing machine $M=$ $\left(Q, \Gamma, \Sigma \supseteq \Gamma, q_{0}, \delta\right)$ with only one tape and time bound $n^{k}$ for some $k \in \mathbb{N}$ that recognizes $L$. Now we use the same construction as in the proof of Theorem 6 . Since $M$ has time bound $n^{k}$ and only a single tape, $M$ has obviously space bound $n^{k}$. So we can describe configurations of $M$ in the very same way as in the proof of Theorem 6 and we can construct a game with positions as before. However, the essential difference here is that at a position $(1, i, q, j, \bar{\delta})$ with $i=n^{k}+2$, the next move does not lead back to the top of $t_{j}^{\bar{\delta}}$, but it leads to the root of a new copy of $t_{j}^{\bar{\delta}}$. If some input $u$ is accepted by $M$, then player 0 can prove this by constructing at most $|u|^{k}$ configurations, so winning strategies carry over between the game constructed in the proof of Theorem 6 and the game constructed here in the obvious way. Moreover, since the graph we have constructed is acyclic by definition, the proof is finished.

\section{Bounded Partial Information}

We turn to the case where the size of the equivalence classes of positions is bounded. The first observation is that bounded tree-width may become unbounded when applying the powerset construction. Afterwards we shall see, that the same result holds for entanglement.

Proposition 11. There are games $\mathcal{G}_{n}, n \in \mathbb{N}$ with bounded partial information and $X\left(\mathcal{G}_{n}\right) \leq 3$ for all $n \in \mathbb{N}$ and any $X \in \mathcal{M}$ such that the corresponding powerset graphs $\bar{G}_{n}$ have unbounded tree-width.

Proof. As a first step consider partial grids, see the second graph on Figure 2 . For an even $n<\omega$, let $\mathfrak{G}_{n}^{1 / 2}$ be obtained from the full, undirected $n \times n$-grid $\mathfrak{G}_{n}$ 
as follows. On each odd horizontal level number $h, h=1,3, \ldots, n-1$ we delete each even vertical edge $(i, h) \longleftrightarrow(i, h+1), i=2,4, \ldots, n$ and on each even horizontal level number $h, h=2,4, \ldots, n-2$ we delete each odd vertical edge $(i, h) \longleftrightarrow(i, h+1), i=1,3, \ldots, n-1$. So altogether we have $\mathfrak{G}_{n}^{1 / 2}=\left(V_{n}, E_{n}^{1 / 2}\right)$ with $(i, j) \stackrel{E}{\longleftrightarrow}(i+1, j)$ for all $1 \leq i \leq n-1$ and all $1 \leq j \leq n$ and $(i, j) \stackrel{E}{\longleftrightarrow}$ $(i, j+1)$ if and only if $i$ and $j$ are both odd or $i$ and $j$ are both even. Similar to the case of the full grids, we have $\operatorname{tw}\left(\mathfrak{G}_{n}^{1 / 2}\right)=n / 2$.

Now we define a class of graphs $G_{n}$ such that the powerset construction converts them to partial grids, see Figure 2. For any even natural number $0<$ $n<\omega$ let $\mathcal{G}_{n}=\left(G_{n}, \sim_{n}^{V}, \sim_{n}^{A}\right)$ where $G_{n}=\left(V_{n}, \emptyset,\left(f_{a}^{n}\right)_{a \in A_{n}}\right)$ is the following game graph:

- $V_{n}=\left\{v_{0}\right\} \cup\{(i, j) \mid 1 \leq i, j \leq n\}$,

- $A_{n}=\left\{a_{i, j} \mid 1 \leq i, j \leq n\right\} \cup\{\leftarrow, \rightarrow\}$,

- $v_{0} \stackrel{a_{i j}}{\longleftrightarrow}(i, j)$ for $1 \leq i, j \leq n$,

$-(i, j) \stackrel{\rightarrow}{\longrightarrow}(i+1, j)$ and $(i+1, j) \stackrel{\leftarrow}{\longrightarrow}(i, j)$ for $1 \leq i \leq n-1$ and $1 \leq j \leq n$.

So $G_{n}$ is a union of $n$ undirected paths, each of length $n$, together with the root $v_{0}$ which has a directed edge to each position $(i, j)$. Obviously, for any measure $X$ we have $X\left(G_{n}\right) \leq 2$.

Partial information is defined as follows. If $i, j \in\{1, \ldots, n\}$ are both odd, then $(i+1, j) \sim_{n}^{V}(i+1, j+1)$ and $a_{i+1, j} \sim_{n}^{A} a_{i+1, j+1}$ and if $i, j \in\{1, \ldots, n\}$ are both even, then $(i-1, j) \sim_{n}^{V}(i-1, j+1)$ and $a_{i-1, j} \sim_{n}^{A} a_{i-1, j+1}$. Notice that with this definition, each equivalence class (of positions as well as of actions) has size at most 2 . Moreover, $\{(1,1)\}$ forms a singleton $\sim_{n}^{V}$-equivalence class.

Now, performing the powerset construction on $\mathcal{G}_{n}$ from $v_{0}$, we obtain the graph $\bar{G}_{v_{0}}^{n}$ which contains an isomorphic copy of $G_{n}$ as a subgraph, where each position $(i, j)$ is replaced by $\{(i, j)\}$. Moreover, for any odd numbers $i, j \in$ $\{1, \ldots, n\}$ we have the position $\{(i+1, j),(i+1, j+1)\}$ from which there are edges to $\{(i, j)\}$ and to $\{(i, j+1)\}$ and for any even number $i, j \in\{1, \ldots, n\}$ we have the position $\{(i-1, j),(i-1, j+1)\}$ from which there are edges to $\{(i, j)\}$ and to $\{(i, j+1)\}$. If $i \neq 1$ and $i \neq n$ there are also other edges from these new vertices (to $\{(i+2, j)\}$ and to $\{(i-2, j)\}$ ), but we do not need to consider them. It is easy to see that $\operatorname{tw}\left(\bar{G}_{v_{0}}^{n}\right) \geq \operatorname{tw}\left(\mathfrak{G}_{n}^{1 / 2}\right)=n / 2$. (Remember that for tree-width we convert directed edges to undirected ones.)

Proposition 12. There are games $\mathcal{G}_{n}, n \in \mathbb{N}$ with bounded partial information and $X(G) \leq 2$ for all $n \in \mathbb{N}$ and any $X \in \mathcal{M} \backslash\{\mathrm{dpw}\}$ such that the corresponding powerset graphs $\bar{G}_{n}$ have unbounded entanglement.

Proof. The graph $G_{n}$ consists of two disjoint copies $\mathcal{T}_{1}$ and $\mathcal{T}_{2}$ of the full undirected binary tree. From a vertex in $\mathcal{T}_{1}$, a directed edge leads to the corresponding vertex in $\mathcal{T}_{2}$ and there are no edges from $\mathcal{T}_{2}$ to $\mathcal{T}_{1}$. Undirected trees have entanglement two, so ent $(G)=2$. The edges from $\mathcal{T}_{1}$ to $\mathcal{T}_{2}$ are implemented by gadgets which create, when the powerset construction is performed, a back edge 

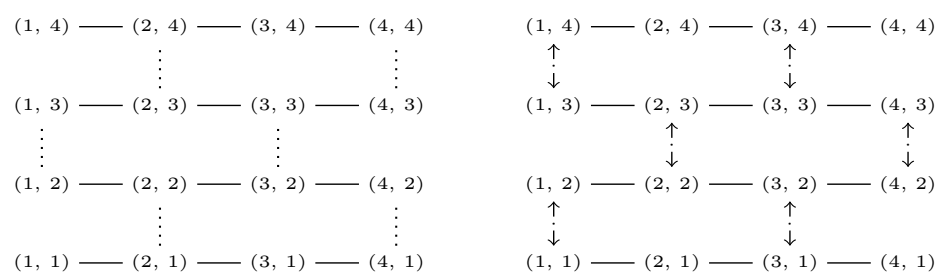

Fig. 2. Game graph $\mathcal{G}_{4}$ (without $v_{0}$ ) and a subgraph of its powerset graph $\bar{G}_{v_{0}}^{4}$

while also preserving the original edge. So the graph $\bar{G}_{n}$ again consists of two disjoint copies of the full undirected binary tree but corresponding vertices are now connected in both directions.

To be more precise, let $n<\omega$ be an even natural number. For an alphabet $\Sigma$ and $k<\omega$, by $\Sigma^{<k}$ we denote the set of all words $u \in \Sigma^{*}$ with $|u|<k$. Furthermore, if $\Sigma$ and $\Gamma$ are alphabets and $\pi: \Sigma \rightarrow \Gamma$ is some function, then for $u=u_{1} \ldots u_{k} \in \Sigma^{*}$, by $\pi(u)$ we denote the word $\pi\left(u_{1}\right) \ldots \pi\left(u_{k}\right) \in \Gamma^{*}$. Now let $\mathcal{G}_{n}=\left(G_{n}, \sim_{n}^{V}, \sim_{n}^{A}\right)$, where $G_{n}=\left(V_{n}, \emptyset,\left(f_{a}^{n}\right)_{a \in A_{n}}\right)$ is the following game graph, see the first graph in Figure 3 By $\pi_{1}$ we denote the mapping $\{0,1\} \rightarrow\{a, b\}$, $0 \mapsto a, 1 \mapsto b$ and by $\pi_{2}$ we denote the mapping $\{a, b\} \rightarrow\{\overline{0}, \overline{1}\}, a \mapsto \overline{0}, b \mapsto \overline{1}$.

$$
\begin{aligned}
& -V_{n}=0\{0,1\}^{<n} \cup a\{a, b\}^{<n} \cup \overline{0}\{\overline{0}, \overline{1}\}^{<n} \\
& -A_{n}=\{0,1, \overline{0}, \overline{1}, \rightarrow, \circlearrowleft\} \\
& -u \stackrel{0}{\longleftrightarrow} u 0 \text { and } u \stackrel{1}{\longleftrightarrow} u 1 \text { for any } u \in 0\{0,1\}^{<n-1} \\
& -u \stackrel{\circlearrowleft}{\longleftrightarrow} u \text { for any } u \in 0\{0,1\}^{<n} . \\
& -u \stackrel{\overline{0}}{\longleftrightarrow} u \overline{0} \text { and } u \stackrel{\overline{1}}{\longleftrightarrow} u \overline{1} \text { for any } u \in \overline{0}\{\overline{0}, \overline{1}\}^{<n-1} \\
& -u \stackrel{\rightarrow}{\longleftrightarrow} \pi_{1}(u) \text { for any } u \in 0\{0,1\}^{<n} . \\
& -u \stackrel{\leftrightarrow}{\longrightarrow} \pi_{2}(u) \text { for any } u \in a\{a, b\}^{<n} .
\end{aligned}
$$

So structurally, $G_{n}$ consists of two disjoint copies of the full undirected binary tree of depth $n$, together with the nodes $u \in a\{a, b\}^{<n}$ which connect the two trees in such a way, that from each $v \in 0\{0,1\}^{<n}$ there is an undirected edge to the corresponding $u \in a\{a, b\}^{<n}$ and there is a directed edge from $u$ to the copy $\bar{v} \in \overline{0}\{\overline{0}, \overline{1}\}^{<n}$ of $v$. It is easy to see that $X\left(G_{n}\right) \leq 3$ for each measure $X$. Partial information is defined by $u \sim_{n}^{V} \bar{u}$ for each $u \in 0\{0,1\}^{<n}, 0 \sim_{n}^{A} \overline{0}$ and $1 \sim_{n}^{A} \overline{1}$.

The powerset construction on $\mathcal{G}_{n}$ from 0 (see Figure 3 yields the graph $\bar{G}_{0}^{n}$ which has $\{0\}$ as position and therefore has also $\{a\}$ and $\{0, \overline{0}\}$ as positions. $\{0\}$ has a directed $\rightarrow$-edge to $\{a\},\{a\}$ has an undirected $\rightarrow$-edge to $\{0, \overline{0}\}$ and, $\{0, \overline{0}\}$ has a directed $\circlearrowleft$-edge back to $\{0\}$. Moreover, $\{0\}$ has an undirected 0-edge to $\{00\}$ and an undirected 1-edge to $\{01\}$. Likewise, $\{0, \overline{0}\}$ has an undirected $(0, \overline{0})$ edge to $\{00, \overline{00}\}$ and an undirected $(1, \overline{1})$-edge to $\{01, \overline{01}\}$. On the lower levels the graph is described completely analog, so it essentially consists of two disjoint copies of the full, undirected binary tree of depth $n$, where each node and its 
duplicate in the other copy are connected by an undirected edge. Adapting a proof from [4] for similar graphs, we now prove ent $\left(\bar{G}_{0}^{n}\right) \geq n / 2-2$.

Assume, the robber is in some leaf node $u \in 0\{0,1\}^{<n}$ such that the unique path from its duplicate $\bar{u} \in \overline{0}\{\overline{0}, \overline{1}\}^{<n}$ to the root position $\overline{0}$ is cop-free. Then, since $u$ has $n-1$ ancestors, but only $n / 2-2$ cops are available to the cop-player, there is some ancestor $v \preceq u$ of $u$ (where $\preceq$ is the prefix order on words) such that the following holds. If $w$ is the predecessor of $v$ in the tree $0\{0,1\}^{<n}$, then $v, \bar{v}, w$ and $\bar{w}$ are cop-free and moreover, from $w$ there is some cop-free path to a leaf node such that also the corresponding duplicate path in the tree $\overline{0}\{\overline{0}, \overline{1}\}^{<n}$ is cop-free. Now the robber moves as follows. He goes from $u$ to $\bar{u}$ and from there, via the cop-free path in $\overline{0}\{\overline{0}, \overline{1}\}^{<n}$, to $\bar{v}$. Notice that the cops can occupy only the vertex where the robber is at the moment. Then he proceeds to $v$, from $v$ to $w$ and from $w$ he goes via the cop-free path in $0\{0,1\}^{<n}$ to a leaf node $u^{\prime} \in 0\{0,1\}^{<n}$. Then, the unique path from its duplicate $\bar{u}^{\prime} \in \overline{0}\{\overline{0}, \overline{1}\}^{<n}$ to the root position $\overline{0}$ is cop-free, so we can use the strategy we have just described again. In this way, the robber is never captured by $n / 2-2$ cops.
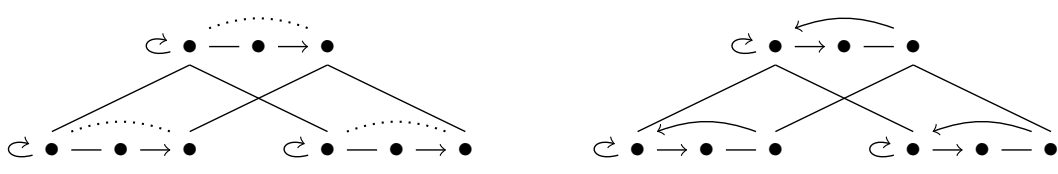

Fig. 3. Game graph $\mathcal{G}_{2}$ and its powerset graph $\bar{G}_{v_{0}}^{2}$.

Cops and robber. Now we prove that in contrast to tree-width and entanglement, non-monotone DAG-width is preserved by the powerset construction. In the following, let $\mathcal{G}=\left(G, \sim^{V}, \sim^{A}\right)$ be a game with bounded partial information, i.e. there is some $r \in \mathbb{N}$ such that $|[u]| \leq r$ for all $u \in V(G)$. Let $\bar{G}$ be the powerset graph of $\mathcal{G}$.

To prove the following results, we first describe the DAG-width game on a graph $G=(V, E)$ in a more formal way. For a set $U \subseteq V$ of vertices we say that a vertex $u^{\prime}$ is reachable from a vertex $u$ in $G-\bar{U}$ if there is a path $u \stackrel{E}{\rightarrow} u^{1} \stackrel{E}{\rightarrow} \ldots \stackrel{E}{\rightarrow} u^{t} \stackrel{E}{\rightarrow} u^{\prime}$ such that $v^{l} \notin U$ for all $l \in\{1, \ldots, t\}$. For sets $U, U^{\prime} \subseteq V$, the set of vertices that are reachable in $G-U$ from some vertex in $U^{\prime}$ is denoted by $\operatorname{Reach}_{G-U}\left(U^{\prime}\right)$. Cops' positions are of the form $(U, v)$ where the cops occupy the set $U$ and the robber is on vertex $v$. The cops can move to any position $\left(U, U^{\prime}, v\right)$, which means that the next set of vertices occupied by the cops will be $U$. The robber's positions are of the form $\left(U, U^{\prime}, v\right)$. The robber can move to any position $\left(U^{\prime}, w\right)$ with $w \notin U^{\prime}$ such that $w$ is reachable from $v$ in $G-\left(U \cap U^{\prime}\right)$. From the initial position, denoted $\perp$, the robber can go to any position $(\emptyset, v)$. So, a play of the DAG-width game with $k$ cops has the form $\pi=\left(U_{0}, v_{0}\right)\left(U_{0}, U_{1}, v_{0}\right)\left(U_{1}, v_{1}\right) \ldots$ where the initial position $\perp$ is omitted and $U_{0}=\emptyset$ such that $\left|U_{i}\right| \leq k$ for all $i$. A play is won by the cop player if it 
finally reaches a position $\left(U, U^{\prime}, v\right)$ such that $\operatorname{Reach}_{G-U \cap U^{\prime}}(v) \subseteq U^{\prime}$. A play is monotonously won by the cop player if it is won by the cop player and never reaches a position $\left(U, U^{\prime}, v\right)$ such that some $u \in U \backslash U^{\prime}$ is reachable from $v$ in $G-U$. Therefore, the DAG-width game has a reachability winning condition and the monotone DAG-width game has a winning condition which is a conjunction of a reachability and a safety condition. Hence, both games are (positionally) determined.

It is easy to see that a cops' strategy only needs to depend on the strongly connected component in which the robber currently is, but not on the particular position within this component. Hence, the cops and robber game can equivalently be described as having positions of the form $(U, R)$ and $\left(U, U^{\prime}, R\right)$ where now, $R$ is a strongly conntected component of $G-U$. From a position $\left(U, U^{\prime}, R\right)$, the robber can move to any position $\left(U^{\prime}, R^{\prime}\right)$ such that $R^{\prime}$ is a strongly connected component of $G-U^{\prime}$ which is reachable from $R$ in $G-\left(U \cap U^{\prime}\right)$.

Proposition 13. If $k$ cops win the DAG-width game on $G$, then $k \cdot r \cdot 2^{r-1}$ cops win the DAG-width game on $\bar{G}$.

Proof. We fisrst describe the proof idea. We translate strategies for $k$ cops from $G$ to $\bar{G}$ and robber's strategies in the opposite direction. Consider positions in games on both graphs. When the robber makes a move on $\bar{G}$ to a vertex $\left\{v_{1}, \ldots, v_{l}\right\}$ we consider $l$ plays in the game on $G$ where he moves to $v_{1}, v_{2}, \ldots$, $v_{l}$. For each of these moves, the strategy for the cops for the game on $G$ supplies an answer, moving the cops from $U$ to $U^{\prime}$. All these moves are translated into a move in which the cops occupy precisely the vertices of $\bar{G}$ that include a vertex from some $U^{\prime}$. These moves of the cop player on $\bar{G}$ can be realized with $k \cdot r \cdot 2^{r-1}$ cops and guarantee that moves of the robber can always be translated back to the game on $G$. The key argument here is that by Lemma 2 , for any path $\bar{u}^{0} \stackrel{\bar{E}}{\longrightarrow} \bar{u}^{1} \stackrel{\bar{E}}{\longrightarrow} \ldots \stackrel{\bar{E}}{\longrightarrow} \bar{u}^{t}$ in $\bar{G}$ and for any $u^{t} \in \bar{u}^{t}$, there is a path $u^{0} \stackrel{E}{\longrightarrow} u^{1} \stackrel{E}{\longrightarrow} \ldots \stackrel{E}{\longrightarrow} u^{t}$ in $G$ such that $u^{i} \in \bar{u}^{i}$ for any $i \in\{0, \ldots, t\}$. So if a play continues infinitely on $\bar{G}$ then at least one corresponding play on $G$ continues infinitely. Hence, if we start from a winning strategy for $k$ cops for the game on $G$, no strategy for the robber can be winning against $k \cdot r \cdot 2^{r-1}$ cops on $\bar{G}$. By determinacy, the result follows.

Formally, let $G=\left(V, V_{0},\left(f_{a}\right)_{a \in A}\right)$ and let $\bar{G}=\left(\bar{V}, \bar{V}_{0},\left(\bar{E}_{a}\right)_{a \in A}\right)$. Let $f$ be a winning strategy for $k$ cops for the DAG-width game on $G$ and let $\bar{g}$ be any strategy for the robber for the DAG-width game on $\bar{G}$. Basically, we translate $f$ to the game on $\overline{\mathcal{G}}$ and $\bar{g}$ in the opposite direction. As vertices in $\bar{G}$ are sets of vertices in $G$, we have to trace multiple plays in $G$ that correspond to one play in $\bar{G}$. Formally, we construct a play $\bar{\pi}_{f g}$ on $\bar{G}$ that is consistent with $\bar{g}$ but not won by the robber. While constructing $\bar{\pi}_{f g}$ we simultaneously construct, for every finite prefix $\bar{\pi}=\left(\bar{U}_{0}, \bar{v}_{0}\right)\left(\bar{U}_{0}, \bar{U}_{1}, \bar{v}_{0}\right)\left(\bar{U}_{1}, \bar{v}_{1}\right) \ldots\left(\bar{U}_{i}, \bar{v}_{i}\right)$ or $\bar{\pi}=$ $\left(\bar{U}_{0}, \bar{v}_{0}\right)\left(\bar{U}_{0}, \bar{U}_{1}, \bar{v}_{0}\right)\left(\bar{U}_{1}, \bar{v}_{1}\right) \ldots\left(\bar{U}_{i-1}, \bar{U}_{i}^{\prime}, \bar{v}_{i-1}\right)$ of $\bar{\pi}_{f g}$, a finite tree $\zeta(\bar{\pi})$ which consists of histories of length $i+1$ in the DAG-width game on $G$, such that the following conditions hold. 
(1) Each history in $\zeta(\bar{\pi})$ is consistent with $f$.

(2) For all $j \leq i+1$ and all $v \in V$ we have $v \in \bar{v}_{j}$ if and only if there is a position $(U, v)$ or $\left(U, U^{\prime}, v\right)$ at level $j+1$ of $\zeta(\bar{\pi})$. Moreover, for each $v \in V$, on each level there is at most one position of the form $(U, v)$ or $\left(U, U^{\prime}, v\right)$.

(3) For all $j \leq i+1$ and all $\bar{u} \in \bar{V}$ we have $\bar{u} \in \bar{U}_{j}$ if and only if there is a position $\left(U^{\prime}, v\right)$ or $\left(U, U^{\prime}, v\right)$ at level $j+1$ of $\zeta(\bar{\pi})$ such that $\bar{u} \cap U^{\prime} \neq \emptyset$.

(4) If $\bar{\pi}^{\prime} \preceq \bar{\pi}$ then $\zeta\left(\bar{\pi}^{\prime}\right) \preceq \zeta(\bar{\pi})$.

Hereby $\bar{\pi}^{\prime} \preceq \bar{\pi}$ means that $\bar{\pi}^{\prime}$ is a prefix of $\bar{\pi}$ and $\zeta\left(\bar{\pi}^{\prime}\right) \preceq \zeta(\bar{\pi})$ means, if $\zeta\left(\bar{\pi}^{\prime}\right)$ has depth $r$ then $\zeta(\bar{\pi})$ has depth $s \geq r$ and up to level $r, \zeta\left(\bar{\pi}^{\prime}\right)$ and $\zeta(\bar{\pi})$ coincide.

To begin the induction consider any history $\bar{\pi}$ of length 1 , i.e. any possible initial move $(\emptyset, \bar{u})$ of the robber player. With $\bar{\pi}$ we associate the tree $\zeta(\bar{\pi})$ consisting of a root $\varepsilon$ which has exactly the positions $(\emptyset, v)$ for $v \in \bar{u}$ as successors. Clearly, conditions (1) - (4) hold. To translate the first cops' move, having a history $\pi=(\emptyset, v)$ (on $G$ ) with $v \in \bar{v}$, consider the set $U_{0}=f(\pi)$ of positions chosen to be occupied by the cops in the first move by the cop player according to $f$. We define $\bar{U}_{0}=\bar{f}(\bar{\pi})$ by $\bar{u} \in \bar{U}_{0}$ if and only if there is some position $(U, v)$ such that $\bar{u} \cap U \neq \emptyset$. This yields the history $\bar{\pi}^{\prime}=\left(\emptyset, \bar{U}_{0}, \bar{v}\right.$ ) (on $\bar{G}$ ). With this history, we associate the tree $\zeta\left(\bar{\pi}^{\prime}\right)$ which is obtained from $\zeta(\bar{\pi})$ by extending each history $\pi=(\emptyset, v)$ in $\zeta(\bar{\pi})$ to $\pi^{\prime}=(\emptyset, v)(\emptyset, f(\pi), v)$. Again, conditions (1) - (4) hold.

For translating the robber's moves in the induction step, consider any history $\bar{\pi}=\left(\bar{U}_{0}, \bar{v}_{0}\right)\left(\bar{U}_{0}, \bar{U}_{1}, \bar{v}_{1}\right)\left(\bar{U}_{1}, \bar{v}_{2}\right) \ldots\left(\bar{U}_{i+1}, \bar{v}_{i+1}\right)$ with $i \geq 1$ and let, by induction hypothesis, $\zeta(\bar{\pi}(\leq i))$ be constructed. The robber has just moved from $\bar{v}_{i}$ to $\bar{v}_{i+1}$, so $\bar{v}_{i+1} \notin \bar{U}_{i}$ and $\bar{v}_{i+1}$ is reachable from $\bar{v}_{i}$ in the graph $\bar{G}-\left(\bar{U}_{i} \cap \bar{U}_{i-1}\right)$. Let $\bar{v}_{i}=\bar{v}^{0} \stackrel{\bar{E}}{\rightarrow} \bar{v}^{1} \stackrel{\bar{E}}{\rightarrow} \ldots \stackrel{\bar{E}}{\rightarrow} \bar{v}^{t}=\bar{v}_{i+1}$ be a path from $\bar{v}_{i}$ to $\bar{v}_{i+1}$ in $\bar{G}-\left(\bar{U}_{i} \cap \bar{U}_{i-1}\right)$. Then, by Lemma 2, for any $v \in \bar{v}_{i+1}$, there is some $u \in \bar{v}_{i}$ such that there is a path $u=u^{0} \stackrel{E}{\rightarrow} u^{1} \stackrel{E}{\rightarrow} \ldots \stackrel{E}{\rightarrow} u^{t}=v$ in $G$ with $u^{l} \in \bar{v}^{l}$ for $l=0, \ldots, t$. By condition (2) for $\zeta(\bar{\pi}(\leq i))$, there is some finite history $\pi \in \zeta(\bar{\pi}(\leq i))$ which ends in a position $\left(U, U^{\prime}, u\right)$, where the last move has been made by the cop player who has chosen $U^{\prime}$ as the new set of positions, occupied by the cops. So, $U$ corresponds to $\bar{U}_{i-1}$ and $U^{\prime}$ corresponds to $\bar{U}_{i}=\bar{U}_{i+1}$ in the sense of condition (3) for $\zeta(\bar{\pi}(\leq i))$. We now extend $\pi$ to the finite history $\pi\left(U^{\prime}, v\right)$. The set of all such histories extended in this way forms the tree $\zeta(\bar{\pi})$. First, we have to show that each such $\pi\left(U^{\prime}, v\right)$ is actually a finite history of the DAG-width game on $G$, i.e. we have to show that $v \notin U^{\prime}$ and $v$ is reachable from $u$ in $G-\left(U \cap U^{\prime}\right)$. First, $\bar{v}_{i+1} \notin \bar{U}_{i}$ and therefore, by condition (3) for $\zeta(\bar{\pi}(\leq i))$ we have $\bar{v}_{i+1} \cap U^{\prime}=\emptyset$ which implies $v \notin U^{\prime}$. Now assume towards a contradiction, that $v$ is not reachable from in $G-\left(U \cap U^{\prime}\right)$. In particular, there must be some $l \in\{1, \ldots, t\}$ such that $u^{l} \in U \cap U^{\prime}$ (notice that $u^{0}=u \notin U \cap U^{\prime}$ ). But then, since $u^{l} \in \bar{v}^{l}$, by $(3)$ for $\zeta(\bar{\pi}(\leq i))$ we have $\bar{v}^{l} \in \bar{U}_{i} \cap \bar{U}_{i-1}$ which contradicts the fact that $\bar{v}^{0} \stackrel{\bar{E}}{\rightarrow} \bar{v}^{1} \stackrel{\bar{E}}{\rightarrow} \ldots \stackrel{\bar{E}}{\rightarrow} \bar{v}^{t}$ is a path in $\bar{G}-\left(\bar{U}_{i} \cap \bar{U}_{i-1}\right)$. Moreover, since all histories in $\zeta(\bar{\pi}(\leq i))$ are compatible with $f$ and all moves by which we have extended histories are made by the robber player, all histories in $\zeta(\bar{\pi})$ are compatible with $f$. Conditions (2), (3) and (4) obviously hold for $\zeta(\bar{\pi})$. 
To translate the cops' answer, for any history $\pi \in \zeta(\bar{\pi})$, consider the set $U=f(\pi)$ of positions chosen to be occupied by the cops in the next move by the cop player according to $f$. We define $\bar{U}=\bar{f}(\bar{\pi})$ by $\bar{v} \in \bar{U}$ if and only if there is some history $\pi \in \zeta(\bar{\pi})$ such that $\bar{v} \cap f(\pi) \neq \emptyset$, i.e. the cops occupy $\bar{v}$ if in some of the plays on $G$ they occupy some vertex in $\bar{v}$. This yields the history $\bar{\pi}^{\prime}=\bar{\pi}\left(\bar{U}_{i+1}, \bar{U}, \bar{v}_{i+1}\right)$. With this history, we associate the tree $\zeta\left(\bar{\pi}^{\prime}\right)$ which is obtained from $\zeta(\bar{\pi})$ by extending each history $\pi \in \zeta(\bar{\pi})$ with last $(\pi)=(U, v)$ to $\pi^{\prime}=\pi(U, f(\pi), v)=\pi\left(U, U^{\prime}, v\right)$. Clearly all the conditions (1) - (4) hold.

Now assume that $\bar{\pi}_{f g}$ is infinite, i.e. won by the robber. Then the tree $\zeta$ which, for any $i<\omega$, coincides up to level $i$ with the tree $\zeta(\bar{\pi}(\leq i))$, is infinite as well. Since $\zeta$ is finitely branching, by König's Lemma there is some infinite path $\pi$ through $\zeta$. By condition (1), $\pi$ is a play in the DAG-width game on $G$ which is compatible with $f$. But since $\pi$ is infinite, this contradicts the fact that $f$ is a winning strategy for the cop player. It remains to count the number of cops used by the cop player in $\bar{\pi}_{f g}$. Consider any position $\left(\bar{U}_{i-1}, \bar{U}_{i}, \bar{v}_{i-1}\right)$ occuring in $\bar{\pi}_{f g}$. By condition (2), at level $i+1$ of $\zeta(\bar{\pi})$, there occur at most $\left|\bar{v}_{i}\right| \leq r$ many histories. Each such history is consistent with $f$, so at most $k$ vertices are occupied by the cops. Hence, by condition (3), $\left|\bar{U}_{i}\right| \leq k \cdot r \cdot 2^{r-1}$. Therefore, the robber does not have a winning strategy against $k \cdot r \cdot 2^{r-1}$ cops in the DAGwidth game on $\bar{G}$. By determinacy, $k \cdot r \cdot 2^{r-1}$ cops have a winning strategy.

Unfortunately, this strategy translation does not necessarily preserve monotonicity, as the following example shows, so boundedness of DAG-width is not implied.

Example 14. We give an example where the strategy translation from Proposition 13 does not preserve monotonicity of the cops' strategy. Consider the graph $G$ depicted in Figure 4 and the following monotone (partial) strategy for the cops. First, put a cop on $v_{0}$. If the robber goes to 1 , put a cop on 1 and then move the cop from 1 to 3 . If the robber goes to 2 , put a cop on 5 and if the robber goes to 4 , put a new cop on 4 . In the game on the powerset graph, consider the following play, which is consistent with the translated cops' strategy. First, the cops occupy $\left\{v_{0}\right\}$. Let the robber go to $\{1,2\}$ in which case the cops occupy $\{1,2\}$ and $\{5\}$. Now the robber goes to $\{3,4\}$, so the cop from $\{1,2\}$ is removed. At this moment, the vertex $\{1,2\}$ becomes available for the robber again, so the translated strategy is non-monotone. Notice that, nevertheless, $\operatorname{dw}(\bar{G})=2$.

Although strategy translation does not preserve monotonicity in the DAGwidth game, an adaptation of the proof of proposition 13 shows that boundedness of directed path-width can be proved by translating strategies non-monotonously. The reason for this is that directed path-width has monotonicity cost 0 .

Proposition 15. If $\operatorname{dpw}(G) \leq k$, then $\operatorname{dpw}(\bar{G}) \leq k \cdot 2^{r-1}$.

Proof. We first make some remarks about the directed path-width game. All the cops know about the possible positions of the robber is what they can deduce from their own actions, namely that the robber is not on some position 

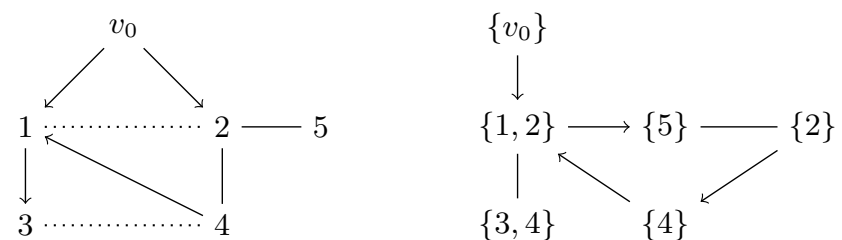

Fig. 4. Monotone strategy is translated to a non-monotone one.

of the graph which has already been searched and has henceforth been blocked for the robber. Positions in this game can be represented as $\left(U, U^{\prime}, R\right)$, where $R \subseteq V$ is a set of vertices such that if the cops move from this position to a position $\left(U^{\prime}, U^{\prime \prime}, R^{\prime}\right)$, then $R^{\prime}=\operatorname{Reach}_{G-\left(U \cap U^{\prime}\right)}(R) \cap\left(V \backslash U^{\prime}\right)$. Basically, this representation is obtained by performing the powerset construction on the original game and then choosing a succinct representation of the positions. Notice that in this representation the moves of the robber player are omitted since they do not change the current position. Strategies can be translated from one representation of the game to the other in the obvious way. Now given a strategy $f$ for the cop player, we obtain a unique play of the game in this new representation of the game which is compatible with $f, \pi_{f}=\left(U_{0}, U_{0}, R_{0}\right)$ $\left(U_{0}, U_{1}, R_{1}\right)\left(U_{1}, U_{2}, R_{2}\right) \ldots$ with $U_{0}=\emptyset, R_{0}=V, U_{i+1}=f(\pi(\leq i))$ and $R_{i+1}=\operatorname{Reach}_{G-\left(U_{i} \cap U_{i+1}\right)}\left(R_{i}\right) \backslash U_{i+1}$.

Let $G=\left(V, V_{0},\left(f_{a}\right)_{a \in A}\right)$ and let $\bar{G}=\left(\bar{V}, \bar{V}_{0},\left(\bar{E}_{a}\right)_{a \in A}\right)$. Let $f$ be a winning strategy for $k$ cops for the directed path-width game on $G$ and let $\pi=$ $\left(U_{0}, U_{0}, R_{0}\right)\left(U_{0}, U_{1}, R_{1}\right) \ldots\left(U_{n-1}, U_{n}, R_{n}\right)$ be the unique play which is compatible with $f$. In order to define the strategy $\bar{f}$ for the cop player for the directed path-width game on $\bar{G}$ it suffices to construct a single maximal finite or infinite sequence $\bar{\pi}=\left(\bar{U}_{0}, \bar{U}_{0}, \bar{R}_{0}\right)\left(\bar{U}_{0}, \bar{U}_{1}, \bar{R}_{1}\right)\left(\bar{U}_{1}, \bar{U}_{2}, \bar{R}_{2}\right) \ldots$ of this game such that $\bar{U}_{0}=\emptyset, \bar{R}_{0}=\bar{V}$ and $\bar{R}_{i+1}=\operatorname{Reach}_{\bar{G}-\left(\bar{U}_{i} \cap \bar{U}_{i+1}\right)}\left(\bar{R}_{i}\right) \backslash \bar{U}_{i+1}$ for all $i$. That means, we construct the strategy $\bar{f}$ by constructing the unique play which is compatible with $\bar{f}$. Then we prove that this play is necessarily finite which shows that the strategy which is defined by this play is a winning strategy for the cops player. For this we inductively define finite histories $\bar{\pi}_{i}=\left(\left(\bar{U}_{0}, \bar{U}_{0}\right), \bar{R}_{0}\right)$ $\ldots\left(\left(\bar{U}_{i-1}, \bar{U}_{i}\right), \bar{R}_{i}\right)$, such that $\bar{U}_{0}=\emptyset, \bar{R}_{0}=\bar{V}$ and for all $j \leq i$, the following conditions hold.

(1) $\bar{R}_{j+1}=\left(\operatorname{Reach}_{\bar{G}-\left(\bar{U}_{j} \cap \bar{U}_{j+1}\right)}\left(\bar{R}_{j}\right)\right) \backslash \bar{U}_{j+1}$.

(2) $\bigcup \bar{R}_{j} \subseteq R_{j}$.

(3) For all $\bar{v} \in \bar{V}$ we have $\bar{v} \in \bar{U}_{j}$ if and only if $\bar{v} \cap U_{j} \neq \emptyset$.

First, $\bar{\pi}_{0}=\left(\bar{U}_{0}, \bar{U}_{0}, \bar{R}_{0}\right)=(\emptyset, \emptyset, \bar{V})$ is already defined by the conditions. Now let $\bar{\pi}_{i}=\left(\bar{U}_{0}, \bar{U}_{0}, \bar{R}_{0}\right)\left(\bar{U}_{0}, \bar{U}_{1} \bar{R}_{1}\right) \ldots\left(\bar{U}_{i-1}, \bar{U}_{i}, \bar{R}_{i}\right)$ be constructed according to the induction hypothesis. If $\bar{R}_{i}=\emptyset$, then the cop player has won so assume that $\bar{R}_{i} \neq$ $\emptyset$. Then $\bigcup \bar{R}_{i} \neq \emptyset$ and by condition (2) this yields that $R_{i} \neq \emptyset$. Therefore, $i<n$. We define $\bar{U}_{i+1}=\left\{\bar{v} \in \bar{V} \mid \bar{v} \cap U_{i+1} \neq \emptyset\right\}$ and $\bar{R}_{i+1}=\operatorname{Reach}_{\bar{G}_{-}-\left(\bar{U}_{i} \cap \bar{U}_{i+1}\right)}\left(\bar{R}_{i}\right) \backslash$ 
$\bar{U}_{i+1}$ which gives us the history $\bar{\pi}_{i+1}=\bar{\pi}_{i}\left(\bar{U}_{i}, \bar{U}_{i+1}, \bar{R}_{i+1}\right)$. Conditions (1) and (3) hold by construction and condition (2) is proved with the same arguments as in the proof of Proposition 13 . Now these finite histories $\bar{\pi}_{i}$ form a prefix-chain $\bar{\pi}_{0} \preceq \bar{\pi}_{1} \preceq \ldots$ which gives us the maximal finite or infinite sequence $\bar{\pi}$. We have already seen in the construction of the histories $\bar{\pi}_{i}$ that if $\bar{\pi}$ was infinite, then $\pi$ would be infinite as well, so $\bar{\pi}$ is in fact a maximal finite history of the directed path-width game on $\bar{G}$ which gives us a winning strategy $\bar{f}$ for the cop player for this game. Moreover, this strategy uses at most $\max \left\{\left|\bar{U}_{i}\right| \mid i=0, \ldots, n\right\}$ cops and for any fixed $i$ we have $\left|\bar{U}_{i}\right| \leq k \cdot 2^{r-1}$. Finally, since directed path-width has monotonicity cost 0 this yields $\operatorname{dpw}(\bar{G}) \leq k \cdot 2^{r-1}$.

Corollary 16. Parity games with bounded partial information can be solved in polynomial time on graphs of bounded directed path-width. If DAG-width has bounded monotonicity cost then the same holds for bounded DAG-width.

An interesting special case where an adapted translation of strategies does preserve monotonicity is given by games with strongly connected equivalence classes of positions. Intuitively this means that for any characteristic of the current state which player 0 is unsure about, it is possible for player 1 to change the value of this characteristic into any other possible value privately, i.e. without changing any characteristics visible for player 0 in between. This is appropriate for situations where, e.g., the uncertainties of player 0 concern some private states of player 1 which are independent of the states visible for player 0 .

Proposition 17. If $\mathrm{dw}(G) \leq k$ and each equivalence class of positions is strongly connected, then $\operatorname{dw}(\bar{G}) \leq k \cdot r^{2} \cdot 2^{r-1}$.

Proof. First, from a monotone winning strategy $h$ for the DAG-width game on $G$ we obtain a monotone winning strategy $f$ for $k \cdot r$ cops for the DAG-width game on $G$ which is compatible with equivalence classes of positions. That means, if $\pi$ is a prefix of a play which is consistent with $f$ and last $(\pi)=\left(U, U^{\prime}, v\right)$, then for any $u \in U^{\prime}$ we have $[u] \subseteq U^{\prime}$ and if $u \in U \backslash U^{\prime}$ then $[u] \cap\left(U \backslash U^{\prime}\right)=\emptyset$. So if the cops occupy a vertex in $G$, then they occupy the whole equivalence class of this vertex. They remain on the whole equivalence class until they would leave every vertex in the class according to $g$. To see that $f$ is monotone assume the opposite, i.e. that there are successive positions $(U, v)$ and $\left(U, U^{\prime}, v\right)$ in a play consistent with $f$ such that there is some $w \in U \backslash U^{\prime}$ which is reachable from $v$ in $G-U$. Then all equivalence classes on the path to $w$ (including $[w]$ ) in the corresponding play according to $h$ were cop free and the robber could reach $w$. However, there was a vertex $w^{\prime}$ in $[w]$ occupied by the cops when playing according to $h$ and after the cops' move in the old game that corresponds to $(U, v) \mapsto\left(U, U^{\prime}, v\right)$, the robber can reach $w^{\prime}$ from $w$ in $[w]$ because $[w]$ is strongly connected. This contradicts the monotonicity of $h$.

Given the strategy $f$, we proceed exactly as in the proof of Proposition 13 It suffices to prove that for each robber's strategy $g$, the play $\bar{\pi}_{f g}$ is not monotonously won by the cops. So assume, towards a contradiction, the opposite, i.e, there is a finite prefix $\bar{\pi} \preceq \bar{\pi}_{f g}$ of $\bar{\pi}_{f g}$ of length $i$ for some $i<\omega$ such that last $(\bar{\pi})=$ 
$\left(\bar{U}, \bar{U}^{\prime}, \bar{v}\right)$ and such that there is some $\bar{u} \in \bar{U} \backslash \bar{U}^{\prime}$ which is reachable from $\bar{v}$ in $\bar{G}-\bar{U}$. So, let $\bar{v}=\bar{v}^{0} \stackrel{\bar{E}}{\rightarrow} \bar{v}^{1} \stackrel{\bar{E}}{\rightarrow} \ldots \stackrel{\bar{E}}{\rightarrow} \bar{v}^{t}=\bar{u}$ be a path from $\bar{v}$ to $\bar{u}$ in $\bar{G}$ with $\bar{v}^{l} \notin \bar{U}$ for $l=0, \ldots, t-1$. Since $\bar{u} \in \bar{U}$ and $\bar{u} \notin \bar{U}^{\prime}$, according to condition (3) for $\zeta(\bar{\pi})$, there is some position $\left(U, U^{\prime}, w\right)$ at level $i+1$ of $\zeta(\bar{\pi})$ such that there is some $u \in \bar{u}$ with $u \in U$ and $u \notin U^{\prime}$. By Lemma 2 there is some $v \in \bar{v}$ such that there is a path $v=v^{0} \stackrel{E}{\rightarrow} v^{1} \stackrel{E}{\rightarrow} \ldots \stackrel{E}{\rightarrow} v^{t}=u$ in $G$ with $v^{l} \in \bar{v}^{l}$ for all $l=0, \ldots, t$. Hence $v^{l} \notin U$ for $l=0, \ldots, t-1$ since if there is some $l \in\{0, \ldots, t-1\}$ such that $v^{l} \in U$, by condition (3) for $\zeta(\bar{\pi})$, we have $\bar{v}^{l} \in \bar{U}$ in contradiction to $\bar{v}^{l} \notin \bar{U}$ for $l=0, \ldots, t-1$. So $u$ is reachable from $v$ in $G-U$. Moreover, by condition (2) for $\zeta(\bar{\pi})$ we have $w \in \bar{v}$ and since $[w]$ is strongly connected, there is a path from $w$ to $v$ in $G$ which is contained in $[w]$. Now $f$ is compatible with equivalence classes of positions and $\bar{v} \notin \bar{U}$, so by condition (3) for $\zeta(\bar{\pi})$ we have $[w] \cap U=\emptyset$. Therefore, $u$ is reachable from $w$ in $G-U$. But since $u \in U \backslash U^{\prime}$ and $\left(U, U^{\prime}, w\right)$ is the last position of a finite history which is compatible with $f$, this contradicts the fact that $f$ is monotone.

Finally, we remark that our direct translation of the robber's moves back to the game on $G$ cannot be immediately applied to the games which define Kellywidth and directed tree-width. In the Kelly-width game, the robber can only move if a cop is about to occupy his vertex. It can happen that the cops occupy a vertex $\left\{v_{1}, \ldots, v_{l}\right\}$ in $\bar{G}$ but not all vertices $v_{1}, \ldots, v_{l}$ in $G$. In the directed tree-width game, the robber is not permitted to leave the strongly connected component in which he currently is, which again obstructs a direct translation of the robber's moves from $\bar{G}$ back to $G$.

\section{New Results}

In this section we introduce new results obtained after the submission of the paper that are related to the results in the published version. Using ideas similar to those from Proposition 17 we prove that DAG-width of the powerset graph obtained from a (directed) graph is a function of the tree-width of the latter.

Cops and splitting robbers. We also introduce the cops and splitting robbers games, which generalize the usual cops and robber games in that now, $k$ cops try to catch (at most) $r$ robbers simultaneously instead of just a single robber. A position in this game has the form $(U, R)$ or $\left(U, U^{\prime}, R\right)$ where $|U|,\left|U^{\prime}\right| \leq k$ and $|R| \leq r$. From a cops' position $(U, R)$, the cops move to any position $\left(U, U^{\prime}, R\right)$ as before. From a robber's position $\left(U, U^{\prime}, R\right)$, the robbers move to any position $\left(U^{\prime}, R^{\prime}\right)$ such that $R^{\prime} \cap U^{\prime}=\emptyset$ and each $r^{\prime} \in R^{\prime}$ is reachable from some $r \in R$ in $G-\left(U \cap U^{\prime}\right)$. That means, the robber player can take robbers from the graph away and he can split robbers into multiple copies which run into different directions such that, after this move, at most $r$ robbers are in the graph. From the initial position $\perp$, the robber player can move to any position $(\emptyset, R)$ with $|R| \leq r$, that means, the first set of vertices occupied by the robbers can be chosen arbitrarily by the robber player. The winning condition of the cops and splitting robber game for the cop play is that finally, all robbers are captured, that means, none 
of the $r$ robbers has a legal move available. Notice that a captured robber can return into the graph on the vertex of another robber who is still in the graph. So a play of the cops and splitting robbers game is won by the cop player if it finally reaches a position $\left(U, U^{\prime}, R\right)$ such that $\operatorname{Reach}_{G-\left(U \cap U^{\prime}\right)}(R) \subseteq U^{\prime}$. Finally the winning condition of the monotone cops and splitting robbers game for the cop player is that, all robbers are captured and additionally, no vertex that has been previously blocked for all robbers becomes reachable for any robber later on. So a play of cops and splitting robbers game is won by the cop player if it finally reaches a position $\left(U, U^{\prime}, R\right)$ such that $\operatorname{Reach}_{G-\left(U \cap U^{\prime}\right)}(R) \subseteq U^{\prime}$ and moreover, it never reaches a position $\left(U, U^{\prime}, R\right)$ such that some $u \in U \backslash U^{\prime}$ is reachable from some $r \in R$ in $G-\left(U \cap U^{\prime}\right)$. Again, a strategy which ensures that such a position is never reached is called monotone. And again, cops and splitting robbers games have reachability conditions and monotone cops and splitting robbers games have winning conditions which are conjunctions of reachability and safety conditions. The minimal $k$ such that $k$ cops have a winning strategy for the monotone cops and $r$ robbers game on $G$ is denoted by $\operatorname{dw}_{r}(G)$.

Eliminating useless moves. In the proof of our second main theorem we use the fact, that any winning strategy for the monotone cops and undirected robber game (or, in fact even in the directed game) can be modified without using additional cops to obtain a strategy that avoid useless moves. That means, the new strategy does not place a cop on a vertex that is already unavailable for the robber. Such move would not make sense and can be skipped, because the strategy is monotone: the robber will never be able to visit the vertex again. Moreover, in any step, the set of positions available for the robber is strictly decreased. This fact is formulated in the following proposition.

Lemma 18. If $\operatorname{tw}(G) \leq k$, then there is a winning strategy $f$ for the cop player for the monotone $k+1$ cops and undirected robber game on $G$, such that for any finite history $\pi$ with last $(\pi)=(U, v)$ we have $f(\pi) \backslash U \neq \emptyset$ and any $u \in f(\pi) \backslash U$ is reachable from $v$ in $G-U$.

Let $U$ be the set of vertices occupied by cops in a game position of the directed game on a graph $G$. The component of the robber is the strongly connected component of $G-U$ with the robber in it. W.l.o.g. we can further assume that $f$ depends only on the strongly connected component of the robber. This is because the robber can still change his vertex in the component before the cops land on their new vertices. So if the robber can reach a next position while cops playing according to such a restricted strategy then he can also reach this position if cops play arbitrary.

Lemma 19. If the cops have a monotone winning strategy in the cops and directed robber game that uses $k$ cops, then they have a positional strategy that depends only on the vertices occupied by cops and on the component of the robber.

We consider the moves of robbers as runs und jumps. A run is a usual move of a single robber along a cop free path. A jump is a move of a robber when he 
leaves his vertex, finds himslef on a vertex $v$ of another robber that the latter occupied in the previous position und possibly runs from $v$ further. In this case we say that robbers $i$ and $j$ split themselves.

Without loss of generality, we restrict the moves of the robbers such that they do not jump to components from which other robbers are jumping in the same move.

Proposition 20. If $\operatorname{tw}(G) \leq k$, then $\operatorname{dw}_{r}(G) \leq r \cdot(k+1)$.

Proof. Let $f$ be a monotone winning strategy for $k$ cops in the undirected game against one robber. We construct a winning monotone strategy $f_{r}$ for $k \cdot r$ cops in the undirected game against $r$ robbers. As the directed game differs from the undirected one only in that the robber is more restricted, $f_{r}$ is monotone and winning for the directed game as well. Notice that the connections between the undirected game and tree-width and between directed game and DAG-width are not symmetrical. If $\operatorname{tw}(G)=k$ then the cop player needs $k+1$ cops to capture the robber in $G$.

In the game against $r$ robbers the cop player uses $r$ teams of cops with $k$ cops in each team. Every team plays independently of each other chasing its own robber according to $f$. To guarantee monotonicity we freeze every team except team 1 and except the teams whose robbers are in the same component as robber 1 and play with them against those robbers. We assume w.l.o.g. that if a robber is captured, he jumps to another one who is not captured yet (if there are some). This guarantees that the cops continue to chase the robbers even if robber 1 is captured. Notice that there may be more than one robber in a component. When a robber $i$ jumps to robber $j$, the positions of its cops are updated to be those of robber $j$.

Formally, a cops' position of the game with $r$ robbers can be described as a tuple $\left(U_{1}, \ldots, U_{r}, R_{1}, \ldots, R_{r}\right)$ where $U_{i}$ is the set of vertices with cops of team $i$ on them and $R_{i}$ is the strongly connected component of $G-\bigcup_{1<i<r} U_{i}$ with robber $i$ in it. Notice that according to Lemma 19, we can assume that $f$ depends only on the vertices occupied by cops and on the component of the robber. We allow multiple cops to occupy the same vertex which is obviously not a restriction. So $f_{r}$ is a function that maps a game position $\left(U_{1}, \ldots, U_{r}, R_{1}, \ldots, R_{r}\right)$ to a tuple $\left(U_{1}^{\prime}, \ldots, U_{r}^{\prime}\right)$.

Let $P:[r] \rightarrow[r]$ be a function that describes the jumps of the robebrs: robber $i$ jumps to robber $P(i)$. (Function $P$ is not uniquely determined, as there may be multiple robbers in the target component. We choose one such function. For convenience, we may assume that $P(i)=P(j)$ if $P(i)$ and $P(j)$ are in the same component, but this is not necessary, because we treat all cops in the component in the same way.) Let $R \subseteq[r]$ be the set of robbers beeing in the same component as robber 1 (including 1 itself) after the robbers move. Then the next move of the cops is

$$
f_{r}\left(U_{1}, \ldots, U_{r}, R_{1}, \ldots, R_{r}\right)=\left(U_{1}^{\prime}, \ldots, U_{r}^{\prime}\right)
$$


where

$$
U_{i}^{\prime}= \begin{cases}f\left(U_{P(i)}, R_{i}\right) & \text { if } i \in R, \\ U_{P(i)} & \text { if } i \notin R .\end{cases}
$$

We have to show that $f_{r}$ is monotone and winning. The following fact can be shown by a straightforward induction on the length of the play.

Fact 1 In every robber's game position, every robber $i$ is guarded only by cops of team $i$ and by cops on vertices where his cops are moving, i.e. $\operatorname{Reach}_{G-\left(U_{i} \cup U_{i}^{\prime}\right)}\left(R_{i}\right) \backslash$ $R_{i}=\emptyset$ and $U_{i}^{\prime} \subseteq U_{P(i)} \cup U_{P(i)}^{\prime}$.

Now we show that no robber $i$ can reach a vertex that has just been left by a cop from team $i$. If the robber did not jump in the previous move, this follows from monotonicity of $f$ and Fact 1 . If the robber jumped in the previous move, then, first, he is bounded by cops of team $P(i)$ and, second, the robbers from $R$ did not jump due to the restriction on the robbers' strategy. So if robber $i$ can reach a vertex $v$ that a cop from team $i$ has left, then $v$ was occupied by a cop of some team from $P(i)$ and is now reachable for a robber from $P(i)$, which is impossible by the first case.

As no robber jumps away from $R_{P(i)}$ for all $i$ and due to Fact 1 . no robber can reach a vertex $v$ that has been left by a cop of another team in the last move. Indeed, if no robber jumped to robber $P(i)$, this follows directly from Fact 1 . If a jumped robber $i$ can reach $v$, then even the robbers in $P(i)$ can reach that vertex because of the second statement of Fact 1 , but this contradicts the monotonicity of $f$.

It remains to show that $f_{r}$ is winning, i.e. that all robbers are finitely captured. We can assume that $f$ does not prescribe to place cops on vertices that are already unavailable for the robber. Such moves would not make sense and can be skipped, because $f$ is monotone: the robber will never be able to visit the vertex again. From this assumption and the monotonicity of $f$ it follows that the set of vertices reachable by robbers is strictly decresasing while the game is played. Thus finally, all robbers are captured.

Conjecture 21. If $\operatorname{dw}(G) \leq k$ and $r \in \mathbb{N}$, then there is a function $F: \mathbb{N}^{2} \rightarrow \mathbb{N}$ such that $\operatorname{dw}_{r}(G) \leq F(k, r)$.

Let $\mathcal{G}=\left(G, \sim^{V}, \sim^{A}\right)$ with $G=\left(V, V_{0},\left(f_{a}\right)_{a \in A}\right.$, col $)$ be a parity game with bounded partial information, that means, there is some $r \in \mathbb{N}$ such that $|[u]| \leq r$ for all $u \in V$.

Proposition 22. If $\mathrm{dw}_{r}(G) \leq k$ then $\mathrm{dw}(\bar{G}) \leq k \cdot 2^{r-1}$.

Proof. Let $G=\left(V, V_{0},\left(f_{a}\right)_{a \in A}\right)$ and $\bar{G}=\left(\bar{V}, \bar{V}_{0},\left(\bar{E}_{a}\right)_{a \in A}\right)$ and let $f$ be a winning strategy for the cop player for $k$ for the monotone cops and $r$ robbers game on $G$. Let moreover $\bar{g}$ be any strategy for the robber player for the montone $k \cdot 2^{r-1}$ cops and (single) robber game on $\bar{G}$. We construct a play $\bar{\pi}_{f g}$ of this game that is consistent with $\bar{g}$ but not won by the robber player. While constructing $\bar{\pi}_{f g}$ we simultaneously construct, for every finite prefix $\bar{\pi}=\left(\bar{U}_{0}, \bar{v}_{0}\right)\left(\bar{U}_{0}, \bar{U}_{1}, \bar{v}_{0}\right)$ 
$\ldots\left(\bar{U}_{i-1}, \bar{U}_{i}, \bar{v}_{i-1}\right)\left(\bar{U}_{i}, \bar{v}_{i}\right)$ or $\bar{\pi}=\left(\bar{U}_{0}, \bar{v}_{0}\right)\left(\bar{U}_{0}, \bar{U}_{1}, \bar{v}_{0}\right) \ldots\left(\bar{U}_{i}, \bar{v}_{i}\right)\left(\bar{U}_{i}, \bar{U}_{i+1}, \bar{v}_{i}\right)$ of $\bar{\pi}_{f g}$, a finite $f$-history $\zeta(\bar{\pi})=\left(U_{0}, \bar{v}_{0}\right)\left(U_{0}, U_{1}, \bar{v}_{0}\right) \ldots\left(U_{i-1}, U_{i}, v_{i-1}\right)\left(U_{i}, \bar{v}_{i}\right)$ or $\zeta(\bar{\pi})=\left(U_{0}, \bar{v}_{0}\right)\left(U_{0}, U_{1}, \bar{v}_{0}\right) \ldots\left(U_{i}, \bar{v}_{i}\right)\left(U_{i}, U_{i+1}, \bar{v}_{i}\right)$ in the cops and $r$ robber game on $G$, such that for all $j \leq i$ we have $\bar{u} \in \bar{U}_{j}$ if and only if $\bar{u} \cap U_{j} \neq \emptyset$. Moreover, if $\bar{\pi}^{\prime}$ is a prefix of $\bar{\pi}$, then $\zeta\left(\bar{\pi}^{\prime}\right)$ is a prefix of $\zeta(\bar{\pi})$.

First, with the history $\bar{\pi}$ which consists only of the initial move $(\emptyset, \bar{u})$ of the robber player, we associate $\zeta(\bar{\pi})=(\emptyset, \bar{u})$. To translate the first cops' move, consider the set $U_{0}=f(\zeta(\pi))$ of positions occupied by the cops in their first move according to $f$. We define $\bar{U}_{0}=\bar{f}(\bar{\pi})$ by $\bar{u} \in \bar{U}_{0}$ if and only if $\bar{u} \cap U_{0} \neq \emptyset$ and with $\bar{\pi}^{\prime}=(\emptyset, \bar{u})\left(\emptyset, \bar{U}_{0}, \bar{u}\right)$ we associate $\zeta\left(\bar{\pi}^{\prime}\right)=(\emptyset, \bar{u})\left(\emptyset, U_{0}, \bar{u}\right)$.

For translating the robber's move in the induction step, consider any history $\bar{\pi}$ $=\left(\bar{U}_{0}, \bar{v}_{0}\right)\left(\bar{U}_{0}, \bar{U}_{1}, \bar{v}_{1}\right)\left(\bar{U}_{1}, \bar{v}_{2}\right) \ldots\left(\bar{U}_{i+1}, \bar{v}_{i+1}\right)$ and let, by induction hypothesis, $\zeta(\bar{\pi}(\leq i))=\left(U_{0}, \bar{v}_{0}\right)\left(U_{0}, U_{1}, \bar{v}_{0}\right)\left(U_{1}, \bar{v}_{1}\right) \ldots\left(U_{i}, U_{i+1}, \bar{v}_{i}\right)$ be constructed. We define $\zeta(\bar{\pi})=\zeta(\bar{\pi}(\leq i))\left(U_{i+1}, \bar{v}_{i+1}\right)$. In the game on $\bar{G}$, the robber has just moved from $\bar{v}_{i}$ to $\bar{v}_{i+1}$, so $\bar{v}_{i+1} \notin \bar{U}_{i+1}$ and $\bar{v}_{i+1}$ is reachable from $\bar{v}_{i}$ in the graph $\bar{G}_{v_{0}}-\left(\bar{U}_{i} \cap \bar{U}_{i+1}\right)$. Let $\bar{v}_{i} \stackrel{\bar{E}}{\rightarrow} \bar{v}^{1} \stackrel{\bar{E}}{\rightarrow} \ldots \stackrel{\bar{E}}{\rightarrow} \bar{v}^{t} \stackrel{\bar{E}}{\rightarrow} \bar{v}_{i+1}$ be a path from $\bar{v}_{i}$ to $\bar{v}_{i+1}$ in $\bar{G}-\left(\bar{U}_{i} \cap \bar{U}_{i+1}\right)$. Now let $v \in \bar{v}_{i+1}$. Then, by Lemma 2, there is some $u \in \bar{v}_{i}$ such that there is a path $u=u^{0} \stackrel{E}{\rightarrow} u^{1} \stackrel{E}{\rightarrow} \ldots \stackrel{E}{\rightarrow} u^{t} \stackrel{E}{\rightarrow} v$ in $G$ with $u^{l} \in \bar{v}^{l}$ for $l=0, \ldots, t$. We have to show that $v \notin U_{i+1}$ and that $v$ is reachable from $u$ in $G-\left(U_{i} \cap U_{i+1}\right)$. First, $\bar{v}_{i+1} \notin \bar{U}_{i+1}$ and therefore, by induction hypothesis for $\zeta(\bar{\pi}(\leq i))$, we have $\bar{v}_{i+1} \cap U_{i+1}=\emptyset$ which implies $v \notin U_{i+1}$. Now assume towards a contradiction, that $v$ is not reachable from $u$ in $G-\left(U_{i} \cap U_{i+1}\right)$. Then there is some $l \in\{1, \ldots, t\}$ such that $u^{l} \in U_{i} \cap U_{i+1}$. But since $u^{l} \in \bar{v}^{l}$, by induction hypothesis for $\zeta(\bar{\pi}(\leq i))$, we have $\bar{v}^{l} \in \bar{U}_{i} \cap \bar{U}_{i+1}$ which contradicts the fact that $\bar{v}^{1} \stackrel{\bar{E}}{\rightarrow} \ldots \stackrel{\bar{E}}{\rightarrow} \bar{v}^{t}$ is a path in $\bar{G}-\left(\bar{U}_{i} \cap \bar{U}_{i+1}\right)$. Therefore, moving the robbers from $\bar{v}_{i}$ to $\bar{v}_{i+1}$ is a legal move for the robber player in the game with $r$ robbers on $G$, so $\zeta(\bar{\pi}$ is an $f$-history with the desired properties.

To translate the cops' answer, consider the set $U=f(\zeta(\bar{\pi}))$ of positions chosen to be occupied by the cops in the next move by the cop player according to $f$. We define $\bar{U}=\bar{f}(\bar{\pi})$ by $\bar{v} \in \bar{U}$ if and only if $\bar{v} \cap U \neq \emptyset$, that means, the cops occupy $\bar{v}$ if in the play on $G$ they occupy some vertex in $\bar{v}$. This yields the history $\bar{\pi}^{\prime}=\bar{\pi}\left(\bar{U}_{i+1}, \bar{U}, \bar{v}_{i+1}\right)$. With this history, we associate the history $\zeta\left(\bar{\pi}^{\prime}\right)=\zeta(\bar{\pi})\left(U_{i+1}, U, \bar{v}_{i+1}\right)$ which clearly has all desired properties.

Now assume that the play $\bar{\pi}_{f g}$ is not monotone, i.e, there is a finite prefix $\bar{\pi} \preceq \bar{\pi}_{f g}$ of $\bar{\pi}_{f g}$ such that last $(\bar{\pi})=\left(\bar{U}_{i}, \bar{U}_{i+1}, \bar{v}_{i}\right)$ is a robber's position and such that there is some $\bar{u} \in \bar{U}_{i} \backslash \bar{U}_{i+1}$ which is reachable from $\bar{v}_{i}$ in $\bar{G}-\bar{U}_{i}$. So, let $\bar{v}_{i} \stackrel{\bar{E}}{\rightarrow} \bar{v}^{1} \stackrel{\bar{E}}{\rightarrow} \ldots \stackrel{\bar{E}}{\rightarrow} \bar{v}^{t} \stackrel{\bar{E}}{\rightarrow} \bar{u}$ be a path from $\bar{v}_{i}$ to $\bar{u}$ in $\bar{G}$ with $\bar{v}^{l} \notin \bar{U}_{i}$ for $l=1, \ldots, t$. Since $\bar{u} \in \bar{U}_{i}$ and $\bar{u} \notin \bar{U}_{i+1}$, by construction of $\zeta(\bar{\pi})$, we have last $(\zeta(\bar{\pi}))=\left(U_{i}, U_{i+1}, \bar{v}_{i}\right)$ and there is some $u \in \bar{u}$ with $u \in U_{i}$ and $u \notin U_{i+1}$. Moreover, by Lemma 2 there is some $v_{i} \in \bar{v}_{i}$ such that there is a path $v_{i} \stackrel{E}{\rightarrow} v^{1} \stackrel{E}{\rightarrow}$ $\ldots \stackrel{E}{\rightarrow} v^{t} \stackrel{E}{\rightarrow} u$ in $G$ with $v^{l} \in \bar{v}^{l}$ for all $l=1, \ldots, t$. Hence $v^{l} \notin U_{i}$ for $l=1, \ldots, t$ since if there is some $l \in\{1, \ldots, t\}$ such that $v^{l} \in U_{i}$ then by construction of $\zeta(\bar{\pi})$, we have $\bar{v}^{l} \in \bar{U}_{i}$ in contradiction to $\bar{v}^{l} \notin \bar{U}$ for $l=1, \ldots, t$. So $u$ is reachable from $v_{i}$ in $G-U_{i}$. But since $v_{i} \in \bar{v}_{i}$ and $u \in U_{i} \backslash U_{i+1}$ and $\left(U_{i}, U_{i+1}, \bar{v}_{i}\right)$ occurs 
in a play which is consistent with $f$, this contradicts the fact that $f$ is strongly monotone.

Now assume that the play $\bar{\pi}_{f g}$ is not monotone, i.e, there is a finite prefix $\bar{\pi} \preceq \bar{\pi}_{f g}$ of $\bar{\pi}_{f g}$ such that last $(\bar{\pi})=\left(\bar{U}_{i}, \bar{U}_{i+1}, \bar{v}_{i}\right)$ is a robber's position and such that there is some $\bar{u} \in \bar{U}_{i} \backslash \bar{U}_{i+1}$ which is reachable from $\bar{v}_{i}$ in $\bar{G}-\bar{U}_{i} \cap \bar{U}_{i+1}$. W.l.o.g. we can assume that there is a path $\bar{v}_{i} \stackrel{\bar{E}}{\rightarrow} \bar{v}^{1} \stackrel{\bar{E}}{\rightarrow} \ldots \stackrel{\bar{E}}{\rightarrow} \bar{v}^{t} \stackrel{\bar{E}}{\rightarrow} \bar{u}$ from $\bar{v}_{i}$ to $\bar{u}$ in $\bar{G}$ with $\bar{v}^{l} \notin \bar{U}_{i}$ for $l=1, \ldots, t$. Since $\bar{u} \in \bar{U}_{i}$ and $\bar{u} \notin \bar{U}_{i+1}$, by construction of $\zeta(\bar{\pi})$, we have last $(\zeta(\bar{\pi}))=\left(U_{i}, U_{i+1}, \bar{v}_{i}\right)$ and there is some $u \in \bar{u}$ with $u \in U_{i}$ and $u \notin U_{i+1}$. Moreover, by Lemma 2 there is some $v_{i} \in \bar{v}_{i}$ such that there is a path $v_{i} \stackrel{E}{\rightarrow} v^{1} \stackrel{E}{\rightarrow} \ldots \stackrel{E}{\rightarrow} v^{t} \stackrel{E}{\rightarrow} u$ in $G$ with $v^{l} \in \bar{v}^{l}$ for all $l=1, \ldots, t$. Hence $v^{l} \notin U_{i}$ for $l=1, \ldots, t$ since if there is some $l \in\{1, \ldots, t\}$ such that $v^{l} \in U_{i}$ then by construction of $\zeta(\bar{\pi})$, we have $\bar{v}^{l} \in \bar{U}_{i}$ in contradiction to $\bar{v}^{l} \notin \bar{U}$ for $l=1, \ldots, t$. So $u$ is reachable from $v_{i}$ in $G-U_{i}$. But since $v_{i} \in \bar{v}_{i}$ and $u \in U_{i} \backslash U_{i+1}$ and $\left(U_{i}, U_{i+1}, \bar{v}_{i}\right)$ occurs in a play which is consistent with $f$, this contradicts the fact that $f$ is strongly monotone.

Now assume that $\bar{\pi}_{f g}$ is won by the robber, i.e., $\bar{\pi}_{f g}$ is infinite. Then the play $\zeta\left(\bar{\pi}_{f g}\right)$ which is obtained by combining all the finite histories $\zeta\left(\bar{\pi}_{f g}(\leq i)\right)$ to last $\left(\left(\zeta\left(\bar{\pi}_{f g}(\leq 0)\right)\right)\right) \ldots$ last $\left(\left(\zeta\left(\bar{\pi}_{f g}(\leq i)\right)\right)\right) \ldots$ for $i<\omega$ is infinite as well. But since each history $\zeta\left(\bar{\pi}_{f g}(\leq i)\right)$ is consistent with $f$, so is the play $\zeta\left(\bar{\pi}_{f g}\right)$ which contradicts the fact that $f$ is a winning strategy for the cop player. Finally, we count the number of cops used by the cops player in $\bar{\pi}_{f g}$. Consider any position $\left(\bar{U}_{i}, \bar{U}_{i+1}, \bar{v}_{i}\right)$ occuring in $\bar{\pi}_{f g}$. Since $\zeta\left(\bar{\pi}_{f g}\right)$ is consistent with $f$, for the corresponding position $\left(U_{i}, U_{i+1}, \bar{v}_{i}\right)$ in $\zeta\left(\bar{\pi}_{f g}\right)$ we have $\left|U_{i+1}\right| \leq k$ and by construction of $\bar{\pi}_{f g}$ it follows that $\left|\bar{U}_{i+1}\right| \leq k \cdot 2^{r-1}$. Therefore, the robber does not have a winning strategy against $k \cdot 2^{r-1}$ cops in the monotone cops and robber game on $\bar{G}$. By determinacy, $k \cdot 2^{r-1}$ cops have a winning strategy.

As for all graphs $G, \operatorname{dw}(G) \leq \operatorname{tw}(G)$, we get the following corollary.

Corollary 23. If $\operatorname{tw}(G) \leq k$, then $\operatorname{dw}(\bar{G}) \leq(k+1) \cdot r \cdot 2^{r-1}$.

Theorem 24. Parity games with bounded partial information played on graphs of bounded tree-width can be solved in polynomial time.

It would be most desirable to obtain this very same result under the weaker assumption that $\operatorname{dw}(G) \leq k$ which would follow immediately from Conjecture 21 . However, Conjecture 21 is much harder to prove than Proposition 20, One main reason for this is the following. In the proof of Proposition 20 we have used the fact that the strategy of the cop player against a single robber does not place cops on vertices which are already unavailable for the robber. This holds for the DAG-width as well. However, it has been essential that the set of vertices which is reachable from a given position is precisely the connected component which contains this positions. So actually we have made heavy use of the fact, that the strategy of the cop player against a single robber does not place cops on vertices outside the robber-component. This does not hold for the DAG-width anymore. More precisely, there is no function $f: \mathbb{N} \rightarrow \mathbb{N}$ such that $\operatorname{dw}(G) \leq k$ implies 
that there is a winning strategy for the cop player for the $f(k)$ cops and directed robber game that does not place cops on vertices outside the robber component. We call cops that can only play ink the robber's component blindfolded.

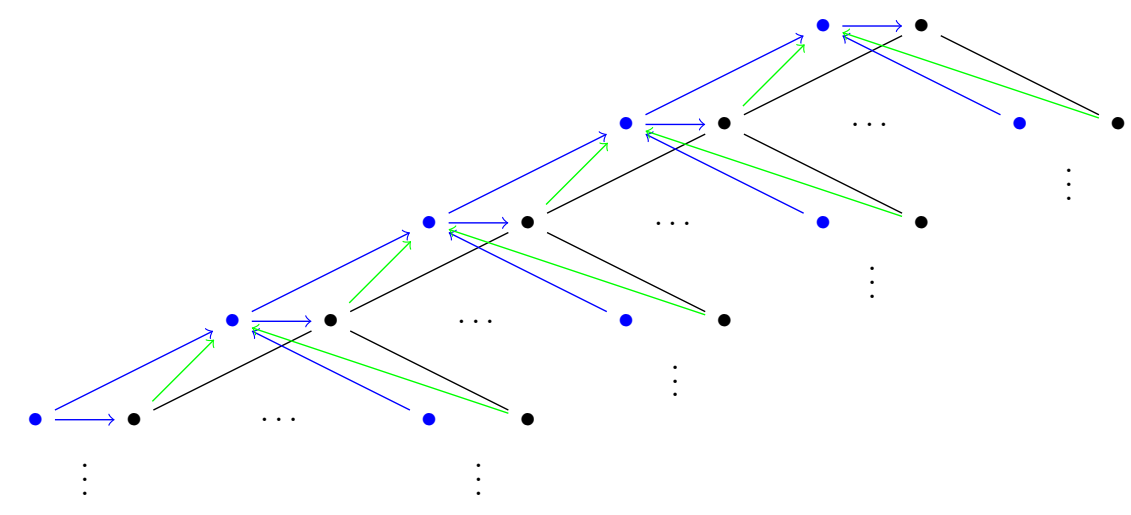

Fig. 5. Four normal, but at least $n+1$ blindfolded cops can capture a robber.

Proposition 25 ([1] $)$. There are games $G_{n}, n \in \mathbb{N}$ such that the following holds.

(1) $\operatorname{dw}\left(G_{n}\right) \leq 4$ for all $n \in \mathbb{N}$

(2) Any winning strategy for the cop player for the blindfolded cops and directed robber game uses at least $n$ cops.

Proof. Consider the following class of directed graphs (see Figure 5). Every graph $G_{n}$ for $0<n<\omega$ is an undirected full tree $\mathcal{T}_{n}=\left(V_{n}, E_{n}\right)$ of degree and depth $n+1$ together with another tree $\mathcal{T}_{n}^{\prime}=\left(V_{n}^{\prime}, E_{n}^{\prime}\right)$ of the same shape with edges directed to the root. Hereby, $V_{n}=\{1, \ldots, n\}^{\leq n+1}$ and $E_{n}$ has edges $\left(\left(v_{1}, \ldots, v_{m}\right),\left(v_{1}, \ldots, v_{m}, v_{m+1}\right)\right)$. Further, $V_{n}^{\prime}=\left\{1^{\prime}, \ldots, n^{\prime}\right\} \leq n+1$ and $E_{n}^{\prime}$ has edges $\left(\left(v_{1}^{\prime}, \ldots, v_{m}^{\prime}\right),\left(v_{1}^{\prime}, \ldots, v_{m}^{\prime}, v_{m+1}^{\prime}\right)\right)$. Additionally, there are edges from any vertex $\left(v_{1}^{\prime}, \ldots, v_{m}^{\prime}\right)$ of the second tree to the corresponding vertex $\left(v_{1}, \ldots, v_{m}\right)$ of the first tree and edges $\left(\left(v_{1}, \ldots, v_{m}\right),\left(v_{1}^{\prime}, \ldots, v_{m}^{\prime}, v_{m-1}^{\prime}\right)\right)$ from every vertex of the first tree to the corresponding parent vertex of the other tree. It is easy to see that four cops capture a robber on every such graph by searching both trees in a top-down manner in parallel. We show that the number of blindfolded cops needed to capture the robber is unbounded.

Let $m$ be a number of cops. Consider the graph $G_{m}$. The robber player has the following winning strategy against $m$ blindfolded cops. At the beginning of a play the robber is on the root $\varepsilon$ where he remains until beeing chased by a cop. At this moment, there is a number $i \in\{1, \ldots, n\}$ such that both subtrees rooted at the roots $i$ and $i^{\prime}$ are cop free. Because of symmetry we can assume that $i=1$. Note that vertex $\varepsilon^{\prime}$ is not in the robber's component and that vertex 
$\varepsilon$ is reachable for the robber, so the cops on $\varepsilon$ is bounded to guard the robber. The robber goes to vertex 1 and waits on 1 until a cop chases him from there. At this moment, there is a number $i$ such that both subtrees rooted at the roots $1 i$ and $1 i^{\prime}$ are cop free. Again we assume that $i=1$. Now vertex $1^{\prime}$ is not in the robber's component. In this way, on level $l$ the robber can bound $l-1$ cops on vertices $1,1^{2}, 1^{3}, \ldots, 1^{l-1}$ and wins when he reaches $1^{n+1}$ because the cops now have to make a non-monotone move.

Notice that we can choose smaller graphs as $G_{n}$, namely those by which the degree of vertices in every level decreases by one.

\section{References}

1. J. Barát. Directed path-width and monotonicity in digraph searching. Graphs and Combinatorics, 22:161-172, 2006.

2. D. Berwanger, A. Dawar, P. Hunter, and S. Kreutzer. DAG-Width and Parity Games. In STACS 06, volume 3884 of $L N C S$, pages 524-536, 2006.

3. D. Berwanger et al. Strategy Construction for Parity Games with Imperfect Information. In CONCUR 08, pages 325-339, 2008.

4. D. Berwanger and E. Grädel. Entanglement - a measure for the complexity of directed graphs with applications to logic and games. In $L P A R$ 2004, volume 3452 of $L N C S$, pages 209-223. Springer, 2005.

5. B. Courcelle. Graph rewriting: An algebraic and logic approach. In Handbook of Theoretical Computer Science, volume B, pages 193-42. Elsevier, 1990.

6. E. Grädel, Ł. Kaiser, and R. Rabinovich. Directed Graphs of Entanglement Two. In FCT 09, volume 5699 of $L N C S$, pages 169-181. Springer, 2009.

7. P. Hunter. Losing the +1 or directed path-width games are monotone. www.comlab.ox.ac.uk/people/paul.hunter/papers/losing.pdf, 2006.

8. P. Hunter and S. Kreutzer. Digraph measures: Kelly decompositions, games, and orderings. In $S O D A$, pages $637-644,2007$.

9. T. Johnson, N. Robertson, P. D. Seymour, and R. Thomas. Directed tree-width. J. Comb. Theory, Ser. B, 82(1):138-154, 2001.

10. M. Jurdziński. Games for Verification: Algorithmic Issues. PhD thesis, University of Aarhus, 2000.

11. Ł. Kaiser, B. Puchala, and R. Rabinovich. Notes on dag-width. Unpublished notes, 2010.

12. S. Kreutzer and S. Ordyniak. Digraph Decompositions and Monotonicity in Digraph Searching. In GTCCS, WG 2008, Durham, pages 336-347, 2008.

13. J. Obdržálek. Algorithmic Analysis of Parity Games. PhD thesis, School of Informatics, University of Edinburgh, 2006.

14. J. Obdržálek. Dag-width: connectivity measure for directed graphs. In SODA, pages 814-821, 2006.

15. B. Puchala. Infinite Two Player Games with Partial Information: Logic and Algorithms. Diploma Thesis, RWTH Aachen, 2008.

16. J. H. Reif. The Complexity of Two-player Games of Incomplete Information. JCSS, 29:274-301, 1984.

17. P. D. Seymour and R. Thomas. Graph searching and a min-max theorem for tree-width. J. Comb. Theory Ser. B, 58(1):22-33, 1993.

18. W. Thomas. On the Synthesis of Strategies in Infinite Games. In STACS '95, pages $1-13,1995$. 
19. K. Wagner and G. Wechsung. Computational Complexity. D. Reidel Publishing Company, 1986. 